\title{
Zeros and Amoebas of Partition Functions
}

\author{
M. Angelelli ${ }^{\mathrm{a})}$ and B. Konopelchenko ${ }^{\mathrm{b}}$ \\ Department of Mathematics and Physics \\ "Ennio De Giorgi", University of Salento and sezione INFN, \\ Lecce, 73100, Italy
}

\begin{abstract}
Singular sectors $\mathcal{Z}_{\text {sing }}$ (loci of zeros) for real-valued non-positively defined partition functions $\mathcal{Z}$ of $n$ variables are studied. It is shown that $\mathcal{Z}_{\text {sing }}$ have a stratified structure and each stratum is a set of certain hypersurfaces in $\mathbb{R}^{n}$. The concept of statistical amoebas is introduced and their properties are studied. Relation with algebraic amoebas is discussed. Tropical limit of statistical amoebas is considered too.
\end{abstract}

Keywords: Partition function, singular sector, amoeba.

\footnotetext{
a)Electronic mail: mario.angelelli@le.infn.it

b)Electronic mail: boris.konopeltchenko@unisalento.it
} 


\section{Introduction}

The partition function is a key object in various branches of physics. In statistical physics, due to the relation with the free energy $F=-k_{B} T \ln \mathcal{Z}$, all basic thermodynamic characteristics of a macroscopic system are encoded in the partition function

$$
\mathcal{Z}=\sum_{n} g_{n} \cdot e^{-\frac{E_{n}}{k_{B} T}}
$$

where $\left\{E_{n}\right\}$ is the energy spectrum, $g_{n}$ is the degeneracy of the $n$-th state, $T$ is the temperature and $k_{B}$ is the Boltzmann constant (see e.g. ${ }^{1,2}$ ). In equilibrium $\mathcal{Z}$ is finite and positive, otherwise it is an indication of instability of the system.

Zeros of the partition function as a function of physical parameters (temperature, magnetic field etc.) are of particular interest since at such points the free energy becomes singular and, hence, the system changes of state, e.g. exhibits a phase transition ${ }^{1,2}$. Since the seminal papers of Lee and Yang ${ }^{3,4}$ it is known that for usual systems (with finite and positive $g_{n}$ and real $E_{n}$ ) zeros of the partition functions lie in the complex plane (see e.g. ${ }^{2,5-14}$ ). These results have led to the intensive study of the partition function's zero sets and associated phase transitions in the complex plane of physical parameters for a number of models in statistical physics, including those subject to quantum dynamics (see e.g. ${ }^{15-18}$ ).

Study of unstable or metastable states is another branch of statistical physics where complex-valued partition functions naturally arise ${ }^{19,20}$. Formally, for an unstable state the energy $E_{n}$ is complex $E_{n}=E_{n, 0}+\mathrm{i} \Delta E_{n}$ with width $\Delta E_{n}$ and, hence, the partition function is complex-valued too. Wide classes of macroscopic systems like spin-glasses and other geometrically or dynamically frustrated systems have such peculiarity (see e.g. ${ }^{21-27}$ ).

In all these cases the situation when partition function's zeros are real is of the greatest interest. The study of properties of macroscopic systems, in particular, structure of equilibrium and unstable domains, is simplified if the partition func- 
tion is real-valued for all values of parameters. Such situation is realisable, for example, for spin-glasses and frustrated systems with different temperatures $T_{n}$ of microsystems (microbasins) if the widths $\Delta E_{n}$ of energy levels obey the condition $\frac{\Delta E_{n}}{k_{B} T_{n}}=\ell_{n} \pi, n=1,2,3, \ldots$ where $\ell_{n}$ are integers. Terms with odd $\ell_{n}$ acquire the factor -1 and, hence, the partition function $\mathcal{Z}$ is of the form (1.1) and real-valued, but with $g_{n}$ assuming both positive and negative values. Negative degeneracies of energy levels can be interpreted as the contribution from sort of holes in spectrum. Formally, negativity of $g_{n}$ is closely connected with the concepts of negative probability and negative membership functions widely discussed in literature (see e.g. ${ }^{28-30}$ and references therein).

This paper is devoted to the study of partition functions of such a type, more precisely, those of the form

$$
\mathcal{Z}(\boldsymbol{g} ; \boldsymbol{x}):=\sum_{\alpha=1}^{N} g_{\alpha} \cdot e^{f_{\alpha}\left(x_{1}, \ldots, x_{n}\right)}
$$

where factors $g_{\alpha}$ take values 1 or $-1, x_{1}, \ldots, x_{n}$ are real variables and $f_{\alpha}(\boldsymbol{x})$ are linear real-valued functions. Main attention is paid to an analysis of the singular sector $\mathcal{Z}_{\text {sing }}$ (locus of zeros) of the partition function (1.2), its stratification and structure of stability $(\mathcal{Z}(\boldsymbol{g} ; \boldsymbol{x})>0)$ and zero confinement domains in the space $\mathbb{R}^{n}$ of parameters $\left(x_{1}, \ldots, x_{n}\right)$.

Singular sector $\mathcal{Z}_{\text {sing }}$ admits a natural stratification

$$
\mathcal{Z}_{\text {sing }}=\bigcup_{k=1}^{\left\lfloor\frac{N}{2}\right\rfloor} \mathcal{Z}_{\text {sing }, k}
$$

the stratum $\mathcal{Z}_{\text {sing, }, k}$ is composed by all hypersurfaces given by $\left(\begin{array}{c}N \\ k\end{array}\right)$ equations

$$
\mathcal{Z}_{k}(\mathcal{I} ; \boldsymbol{x}):=-\sum_{\alpha \in \mathcal{I}} e^{f_{\alpha}(\boldsymbol{x})}+\sum_{\beta \notin \mathcal{I}} e^{f_{\beta}(\boldsymbol{x})}=0, \quad k=1, \ldots,\left\lfloor\frac{N}{2}\right\rfloor
$$

where $(\mathcal{I},[N] \backslash \mathcal{I})$ is any 2-partition of the set $[N]:=\{1,2, \ldots, N\}$ with cardinality $\# \mathcal{I}=k$. These hypersurfaces for the $k$-stratum are contained in a certain domain 
in $\mathbb{R}^{n}$ refered as the zero confinement domain $Z C D_{k}$. This domain is divided by hypersurfaces (1.4) into a number of subdomains $Z C D_{k ; \delta}$ at which each of the $\left(\begin{array}{l}N \\ k\end{array}\right)$ functions $\mathcal{Z}_{k}$ in (1.4) is positive or negative. This allows to associate with each of these subdomains a set of $\left(\begin{array}{l}N \\ k\end{array}\right)$ number 1 or -1 that can be viewed as the state of the system of $\left(\begin{array}{l}N \\ k\end{array}\right)$ "spins" which take values 1 or -1 .

The domain $\mathcal{D}_{k+}$, where all functions (1.4) are positive, is the stability (equilibrium) domain. The union $\mathcal{A}_{k}:=\mathcal{D}_{k+} \cup Z C D_{k}$ is called the statistical $k$-amoeba. A $k$-statistical amoeba is composed by the stable nucleus $\mathcal{D}_{k+}$ and intermittent shell $Z C D_{k}$ with varying degree of instability (number of signs -1 ). The complement $\mathcal{D}_{k-}$ of the $k$-amoeba in $\mathbb{R}^{n}$ is the domain with maximum number of signs -1 , i.e. the domain of maximal instability.

The domains $\mathcal{D}_{k+}, Z C D_{k}$ and statistical $k$-amoebas exhibit a simple inclusion property, for instance, $\mathcal{D}_{k+} \supseteq \mathcal{D}_{\hat{k}+}$ and $\mathcal{A}_{k} \supseteq \mathcal{A}_{\hat{k}}$ if $1 \leq k<\hat{k}<\frac{N}{2}$.

Statistical 1-amoebas $\mathcal{A}_{1}$ coincide with the so-called non-lopsided amoebas $\mathcal{L} \mathcal{A}$ introduced in algebraic geometry ${ }^{31}$. Analogs of higher statistical $k$-amoebas $(k \geq$ 2) seem to be not studied in algebraic geometry.

Tropical limits of statistical $k$-amoebas are considered. It is shown that all $k$ amoebas collapse into the same set of piecewise hyperplanes in $\mathbb{R}^{n}$ coinciding with that of tropical limit of $\mathcal{A}_{1}$.

It should be noted that partition functions depending on several variables (multidimensional energy spectrum or several Hamiltonians) have been considered in $^{32}, 33$ and $^{34}$. However these papers have addressed only the case (1.2) with all positive factors $g_{\alpha}$.

In a completely different setting zeros of superpositions of the form (1.2) $(\tau$ functions) with positive and negative factors $g_{\alpha}$ and very particular linear functions $f_{\alpha}$ arise within an analysis of singular solutions of integrable equations (see e.g. $\left.{ }^{35},{ }^{36}\right)$.

The paper is organized as follows. General definitions of strata of the singular 
sector and some concrete examples for the first stratum $\mathcal{Z}_{\text {sing, } 1}$ are given in section 2. Higher strata and properties of zero loci hypersurfaces are considered in section 3. Section 4 is devoted to the study of some general properties of equilibrium and zero confinement domains. Statistical amoebas and their relation to algebraic amoebas are discussed in section 5. Next section 6 is devoted to an analysis of the structure and properties of $Z C D_{k}$ domains and associated statistical systems of "spins". Tropical limits of statistical amoebas are considered in section 7. In conclusion some peculiarities of partition function (1.2) with nonlinear functions $f_{\alpha}$ are noted.

\section{Singular sector of partition function}

So we will consider the family of partition functions of the form

$$
\mathcal{Z}=\sum_{\alpha=1}^{N} g_{\alpha} \cdot e^{f_{\alpha}(\boldsymbol{x})}
$$

where $f_{\alpha}(\boldsymbol{x})=b_{\alpha}+\sum_{i=1}^{n} a_{\alpha i} x_{i}$, all variables $g_{\alpha}, x_{i}$ and all functions $f_{\alpha}$ are real. Since $g_{\alpha} e^{b_{\alpha}}=\operatorname{sign}\left(g_{\alpha}\right) \cdot e^{\log \left|g_{\alpha}\right|+b_{\alpha}}$ one can consider only the case $g_{\alpha} \in\{+1,-1\}$.

The space $V$ of parameters $g_{1}, \ldots, g_{N}, x_{1}, \ldots, x_{n}$ admits the stratification

$$
V=\bigcup_{\alpha=0}^{N} V_{\alpha}
$$

where $V_{\alpha}$ is the union of subspaces of $V$ with $\alpha$ many negative $g_{\beta}$. For instance, $V_{2}=\bigcup_{1 \leq \alpha<\beta \leq N,} V_{2,\{\alpha \beta\}}$ where $V_{2,\{\alpha \beta\}}=\left\{\left(g_{1}, \ldots, g_{N} ; x_{1}, \ldots, x_{n}\right): g_{\alpha}=g_{\beta}=-1\right.$, $\left.\gamma \neq \alpha, \beta \Rightarrow g_{\gamma}>0\right\}, \alpha \neq \beta, \alpha, \beta=1, \ldots, N$. Inversion $P$ of all $g_{\alpha}: P g_{\alpha}=-g_{\alpha}$, $\alpha=1, \ldots N$, acts on strata $V_{\alpha}$ as $P V_{\alpha}=V_{N-\alpha}$.

Accordingly, singular sector $\mathcal{Z}_{\text {sing }}$ of partition function also admits the stratification

$$
\mathcal{Z}_{\text {sing }}=\bigcup_{k=1}^{N-1} \mathcal{Z}_{\text {sing }, k}
$$


where $\mathcal{Z}_{\text {sing, } k}$ are subspaces of $V_{k}$ for which $\left.\mathcal{Z}\right|_{V_{k}}=0$. Subspaces $V_{0}$ and $V_{N}$ are obviously regular and connected by inversion $P$. First singular stratum $\mathcal{Z}_{\text {sing, } 1}$ is the union of the $N$ hypersurfaces defined by the equations

$$
\mathcal{Z}_{1}(\{\alpha\} ; \boldsymbol{x}):=\sum_{\beta=1}^{N} g_{\alpha(\beta)} e^{f_{\beta}(\boldsymbol{x})}=0, \quad \alpha=1, \ldots, N
$$

with $g_{\alpha(\alpha)}=-1$ and $g_{\alpha(\beta)}=1, \beta \neq \alpha$. Geometric characteristics of such hypersurfaces have been studied in the paper ${ }^{37}$. Cases of linear functions $f_{\alpha}(\boldsymbol{x})$ were referred in $^{37}$ as ideal statistical hypersurfaces. General statistical hypersurfaces considered in $^{37}$ were associated with nonlinear functions $f_{\alpha}$ while super-ideal case corresponds to $N=n$ and $f_{\alpha}(\boldsymbol{x}) \equiv x_{\alpha}$.

Generically the stratum $\mathcal{Z}_{\text {sing, } 1}$ can be composed by $N$ hypersurfaces. In addition, it is easy to see that hypersurfaces given by $\mathcal{Z}_{1}(\{\alpha\} ; \boldsymbol{x})=0$ with different $\alpha$ do not intersect at finite values of $x_{1}, \ldots, x_{n}$ and $N \geq 3$. Indeed, if there exists $\alpha \neq \beta$ such that $\left\{\boldsymbol{x}: \mathcal{Z}_{1}(\{\alpha\} ; \boldsymbol{x})=0\right\} \cap\left\{\boldsymbol{x}: \mathcal{Z}_{1}(\{\beta\} ; \boldsymbol{x})=0\right\}$ is not empty, then there exists $\boldsymbol{x}_{0}$ in $\mathbb{R}^{n}$ such that $e^{f_{\beta}\left(\boldsymbol{x}_{0}\right)}-e^{f_{\alpha}\left(\boldsymbol{x}_{0}\right)}=\sum_{\gamma \notin\{\alpha, \beta\}} e^{f_{\gamma}\left(\boldsymbol{x}_{0}\right)}=e^{f_{\alpha}\left(\boldsymbol{x}_{0}\right)}-e^{f_{\beta}\left(\boldsymbol{x}_{0}\right)}$, that is $f_{\beta}\left(\boldsymbol{x}_{0}\right)=f_{\alpha}\left(\boldsymbol{x}_{0}\right)$, so one has $\sum_{\gamma \neq \alpha, \beta} e^{f_{\gamma}\left(\boldsymbol{x}_{0}\right)}=0$ which is impossible if $N \geq 3$.

The form of hypersurfaces which compose the sector $\mathcal{Z}_{\text {sing, } k}$ depends on $N, n$ and the choice of functions $f_{\alpha}(\boldsymbol{x})$. At the simplest case of $n=1$ the stratum $\mathcal{Z}_{\text {sing, } 1}$ is composed, in general, by at most $N$ points defined by the equations

$$
\mathcal{Z}_{1}\left(\{\alpha\} ; x_{1}\right)=\sum_{\beta=1}^{N} g_{\alpha(\beta)} e^{a_{\beta} x_{1}+b_{\beta}}=0, \quad \alpha=1, \ldots, N
$$

with $g_{\alpha(\alpha)}=-1$ and $g_{\alpha(\beta)}=1, \beta \neq \alpha$. In the case $N=2$ the sector $\mathcal{Z}_{\text {sing, } 1}$ contains only one hypersurface defined by the equation

$$
\mathcal{Z}_{1}(\{1\} ; \boldsymbol{x})=-e^{f_{1}\left(x_{1}, \ldots, x_{n}\right)}+e^{f_{2}\left(x_{1}, \ldots, x_{n}\right)}=0 .
$$

It is the hyperplane in $\mathbb{R}^{n}$ given by the equation

$$
b_{1}-b_{2}+\sum_{i=1}^{n}\left(a_{1 i}-a_{2 i}\right) x_{i}=0 .
$$


For $N \geq 3$ and $n=2$ one has a family of curves on the plane $\left(x_{1}, x_{2}\right)=:(x, y)$. For example, at the choice $f_{1} \equiv 0, f_{2} \equiv x, f_{3} \equiv y$ the sector $\mathcal{Z}_{\text {sing, }, 1}$ is composed by three curves shown in figure 1 (a) $\left(\right.$ see also ${ }^{46}$ ) where the curves 1,2 and 3 are

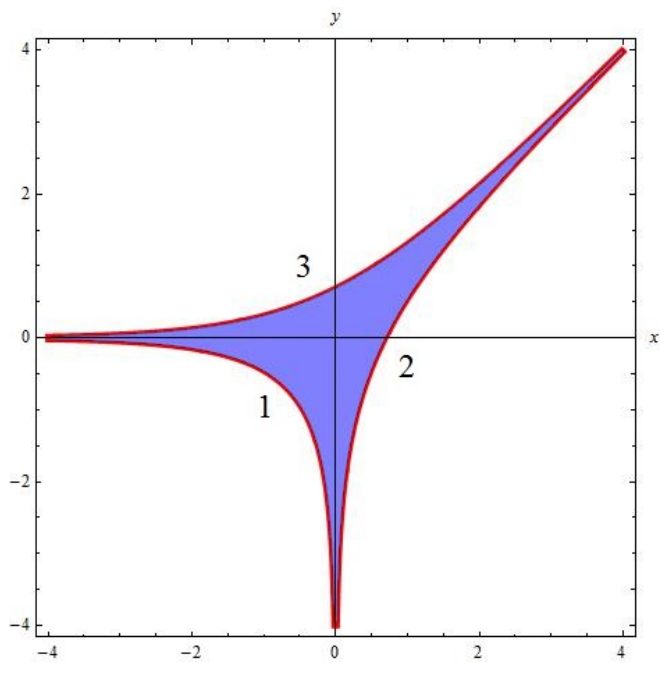

(a) $f_{1} \equiv 0, f_{2} \equiv x, f_{3} \equiv y$.

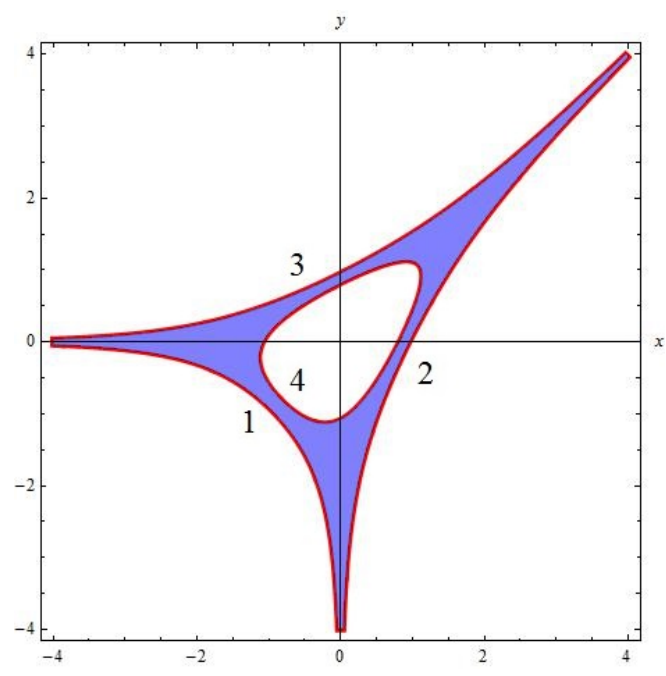

(b) $f_{1} \equiv 0, f_{2} \equiv 3 x, f_{3} \equiv 3 y$,

$$
f_{4} \equiv x+y+\ln 6 .
$$

Figure 1. Examples of 1-strata (red curves).

given by the equations

$$
\begin{gathered}
\mathcal{Z}_{1}(\{1\} ; x, y) \equiv-1+e^{x}+e^{y}=0, \\
\mathcal{Z}_{1}(\{2\} ; x, y) \equiv 1-e^{x}+e^{y}=0, \\
\mathcal{Z}_{1}(\{3\} ; x, y) \equiv 1+e^{x}-e^{y}=0 .
\end{gathered}
$$

Note that at $|x|,|y| \rightarrow \infty$ the curves 1 and 2 tend to the ray $x=0, y<0$, the curves 2 and 3 tend to the ray $x=y, x>0$ while the curves 3 and 1 tend to the ray $y=0, x<0$. An example with a different homotopy and a bounded closed component (curve 4 ) is given by choice $f_{1} \equiv 0, f_{2} \equiv 3 x, f_{3} \equiv 3 y, f_{4} \equiv x+y+\ln 6$ and it is shown in figure 1(b).

The stratum $\mathcal{Z}_{\text {sing, } 1}$ at $n=2$ is composed by at most $N$ curves. For particular choice of functions $f_{\alpha}(\boldsymbol{x})$ this number can be smaller than $N$ when some equa- 
tions in (2.4) define the empty set. To illustrate this let us consider the following examples with $n=2$ : if one chooses

$$
f_{\alpha}(x, y) \equiv \cos \left(\frac{2 \pi(\alpha-1)}{N}\right) \cdot x+\sin \left(\frac{2 \pi(\alpha-1)}{N}\right) \cdot y, \quad \alpha=1, \ldots, N
$$

then one gets $N$ curves by symmetry. For instance, at $N=6$ one gets 6 curves presented in figure 2(a). If, instead, one takes

$$
f_{\alpha}(x, y) \equiv(\alpha-1) \cdot x+(N-\alpha) \cdot y, \quad \alpha=1, \ldots, N
$$

then for all $2 \leq \gamma \leq N-1$ the corresponding locus is empty, i.e. $\left\{\mathcal{Z}_{1}(\{\gamma\})=\right.$ $0\}=\emptyset$. Indeed, if $x \leq y$ then $e^{(\gamma-1) \cdot x} \leq e^{(\gamma-1) \cdot y}$ thus $e^{f_{\gamma}(x, y)} \leq e^{f_{1}(x, y)}<\sum_{\beta \neq \gamma} e^{f_{\beta}(x, y)}$. Similarly, If $y \leq x$ then $e^{f_{\gamma}(x, y)} \leq e^{f_{N}(x, y)}<\sum_{\beta \neq \gamma} e^{f_{\beta}(x, y)}$. The only two visible curves are given by $\mathcal{Z}_{1}(\{1\} ; x, y)=0$ and $\mathcal{Z}_{1}(\{N\} ; x, y)=0$ : they are straight lines with slope 1 passing through $( \pm \ln \chi, 0)$ respectively, where $\chi>0$ is uniquely defined by $-1+\sum_{\alpha=1}^{5} \chi^{\alpha}=0$. See figure $2(\mathrm{~b})$ for the case at $N=6$.

One has an intermediate case at $f_{1}(x, y) \equiv 0, f_{2}(x, y) \equiv 3 x, f_{3}(x, y) \equiv 3 y$, $f_{4}(x, y) \equiv x+y+\ln 6, f_{5}(x, y) \equiv 2 x+y+\ln 11, f_{6}(x, y) \equiv x+3 y+\ln 4$. The stratum $\mathcal{Z}_{\text {sing, } 1}$ is composed by 5 curves given in figure 3 . One can check directly that the set of solutions of the equation

$$
\mathcal{Z}_{1}(\{4\} ; x, y) \equiv 1+e^{3 x}+e^{3 y}-6 \cdot e^{x+y}+11 \cdot e^{2 x+y}+4 e^{x+3 y}=0
$$

is empty. Indeed, if $\mathcal{Z}_{1}(\{4\} ; x, y)=0$ then $x+y+\ln 6>2 x+y+\ln 11$, that is $x<\ln 6-\ln 11<0$. From the arithmetic-geometric means inequality one gets $\frac{1}{2}+\frac{1}{2}+11 \cdot e^{2 x+y}+4 \cdot e^{x+3 y} \geq 4\left[\frac{1}{2} \cdot \frac{1}{2} \cdot\left(11 \cdot e^{2 x+y}\right) \cdot\left(4 \cdot e^{x+3 y}\right)\right]^{\frac{1}{4}}=4 \cdot 11^{\frac{1}{4}} \cdot e^{\frac{3 x+4 y}{4}}$. But $4 \cdot 11^{\frac{1}{4}}>6$ and $x<0$, hence $e^{\frac{3 x+4 y}{4}}>e^{x+y}$ and $1+11 \cdot e^{2 x+y}+4 \cdot e^{x+3 y} \geq$ $4 \cdot 11^{\frac{1}{4}} \cdot e^{\frac{3 x+4 y}{4}}>4 \cdot 11^{\frac{1}{4}} \cdot e^{x+y}>6 \cdot e^{x+y}$. In particular, $\mathcal{Z}_{1}(\{4\} ; x, y)$ is always positive.

At $N=3, n=3$ and $f_{1} \equiv x, f_{3} \equiv y, f_{4} \equiv z\left(x_{1}=x, x_{2}=y, x_{3}=z\right)$ the 


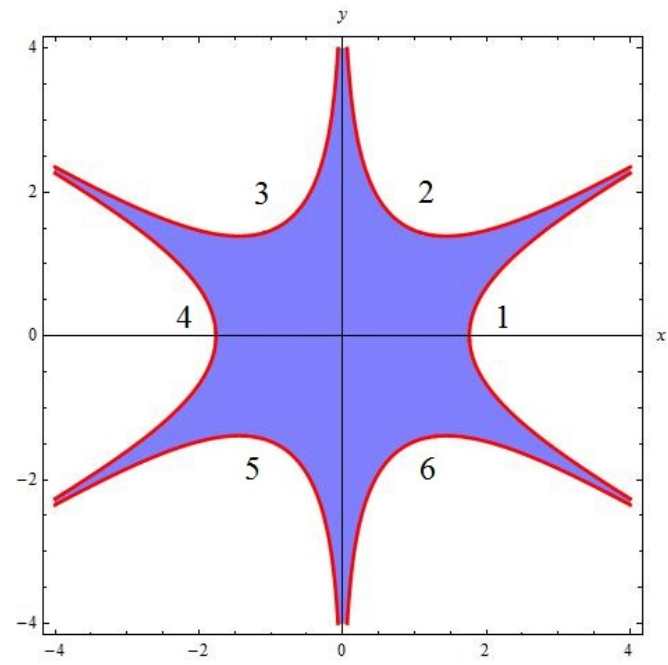

(a) $f_{\alpha} \equiv \cos \left(\frac{\pi(\alpha-1)}{3}\right) x+\sin \left(\frac{\pi(\alpha-1)}{3}\right) y$, $\alpha=1, \ldots 6$ : all 6 components are visible.

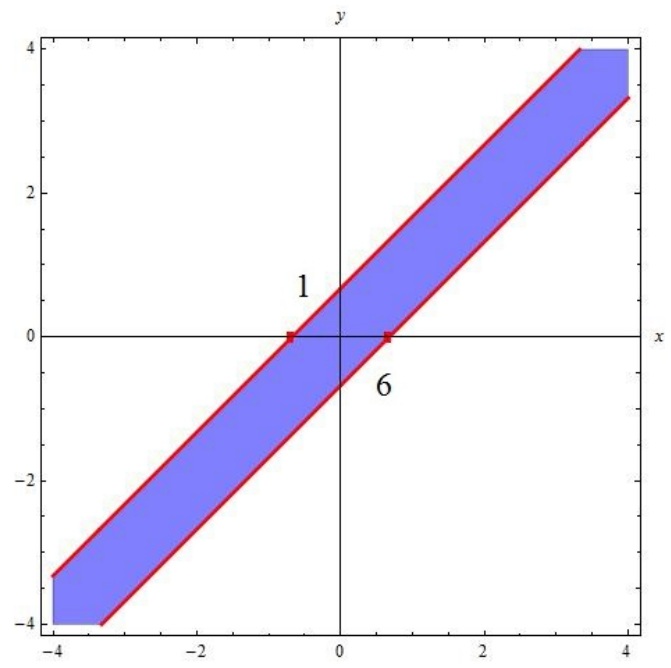

(b) $f_{\alpha}(x, y):=(\alpha-1) \cdot x+(6-\alpha) \cdot y$, $\alpha=1, \ldots, 6$.

Figure 2. Extremal behaviors of number of visible curves.

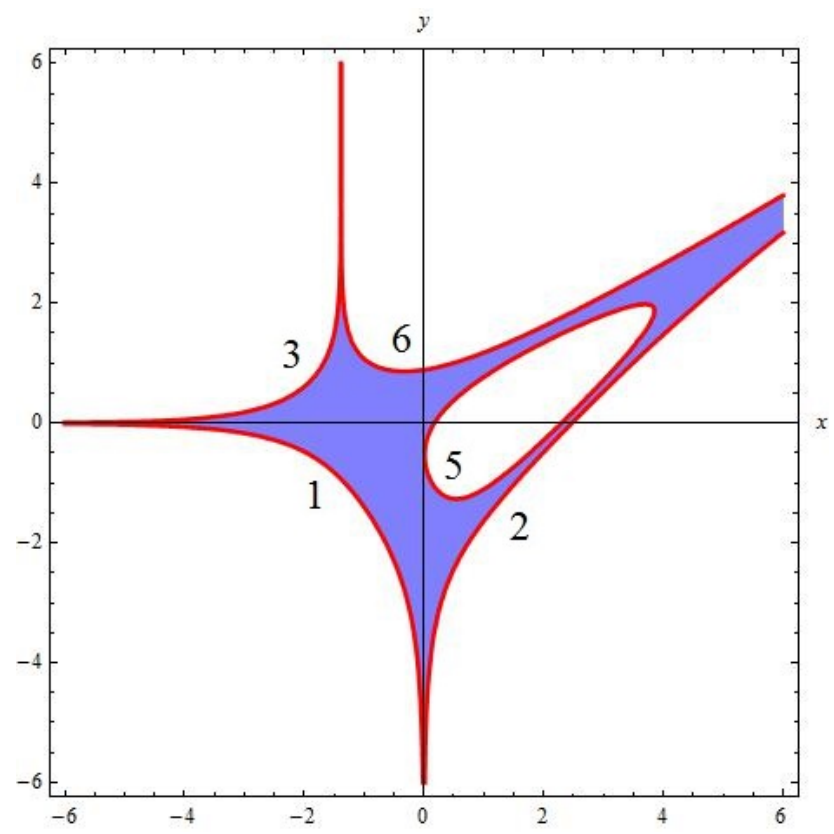

Figure 3. 1-stratum for the choice $f_{1}(x, y) \equiv 0, f_{2}(x, y) \equiv 3 x, f_{3}(x, y) \equiv 3 y, f_{4}(x, y) \equiv$ $x+y+\ln 6, f_{5}(x, y) \equiv 2 x+y+\ln 11, f_{6}(x, y) \equiv x+3 y+\ln 4$. 
stratum $\mathcal{Z}_{\text {sing, } 1}$ contains 3 super-ideal statistical surfaces defined by the equations

$$
\begin{gathered}
\mathcal{Z}_{1}(\{1\} ; x, y, z) \equiv-e^{x}+e^{y}+e^{z}=0 \\
\mathcal{Z}_{1}(\{2\} ; x, y, z) \equiv e^{x}-e^{y}+e^{z}=0 \\
\mathcal{Z}_{1}(\{3\} ; x, y, z) \equiv e^{x}+e^{y}-e^{z}=0
\end{gathered}
$$

and given in figure 4 . Induced metric $g_{i k}$, Gauss curvature $K$ and mean curvature

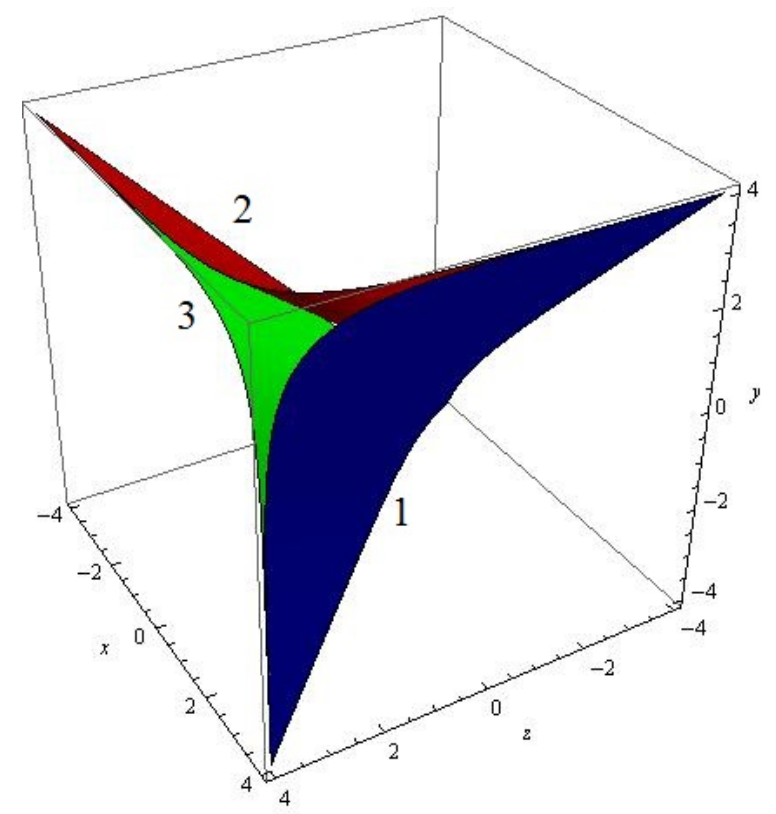

Figure 4. 1-stratum for the super-ideal case: $f_{1}(x, y, z) \equiv x f_{2}(x, y, z) \equiv y, f_{3}(x, y, z) \equiv$ $z$.

$\Omega$ of the surface given by the equation $\mathcal{Z}_{1}(\{1\})=0$ are (with $y$ and $z$ as local (coordinates) $)^{37}$

$$
\begin{gathered}
g_{i k}=\delta_{i k}+w_{i} w_{k}, \quad i, k=1,2, \\
K=0 \\
\Omega=\frac{1-T_{3}}{\left(1+T_{2}\right)^{\frac{3}{2}}}
\end{gathered}
$$

where probability $w_{1}=\frac{e^{y}}{e^{y}+e^{z}}, w_{2}=\frac{e^{z}}{e^{y}+e^{z}}$ and $T_{l}=w_{1}^{l}+w_{2}^{l}, l=2,3$. For surface given by the equation $\mathcal{Z}_{1}(\{2\})=0$ and $\mathcal{Z}_{1}(\{3\})=0$ one has similar results 
with substitution $x \leftrightarrows y$ and $x \leftrightarrows z$, respectively. At large $x, y, z$ surfaces 1 and 2 tend to the half-plane $x=y, z<0$, surfaces 1 and 3 tend to the half-plane $x=z$, $y<0$ and surfaces 2 and 3 tend to the half-plane $y=z, x<0$.

Last example is presented in figure 5 and corresponds to $N=6$ and $n=3$ with $f_{1}(x, y, z)=0, f_{2}(x, y, z)=3 x, f_{3}(x, y, z)=3 y, f_{4}(x, y, z)=2 x+z+\log 6$, $f_{5}(x, y, z)=2 x+y+z+\log 11, f_{6}(x, y, z)=3 y+z+\log 4$.

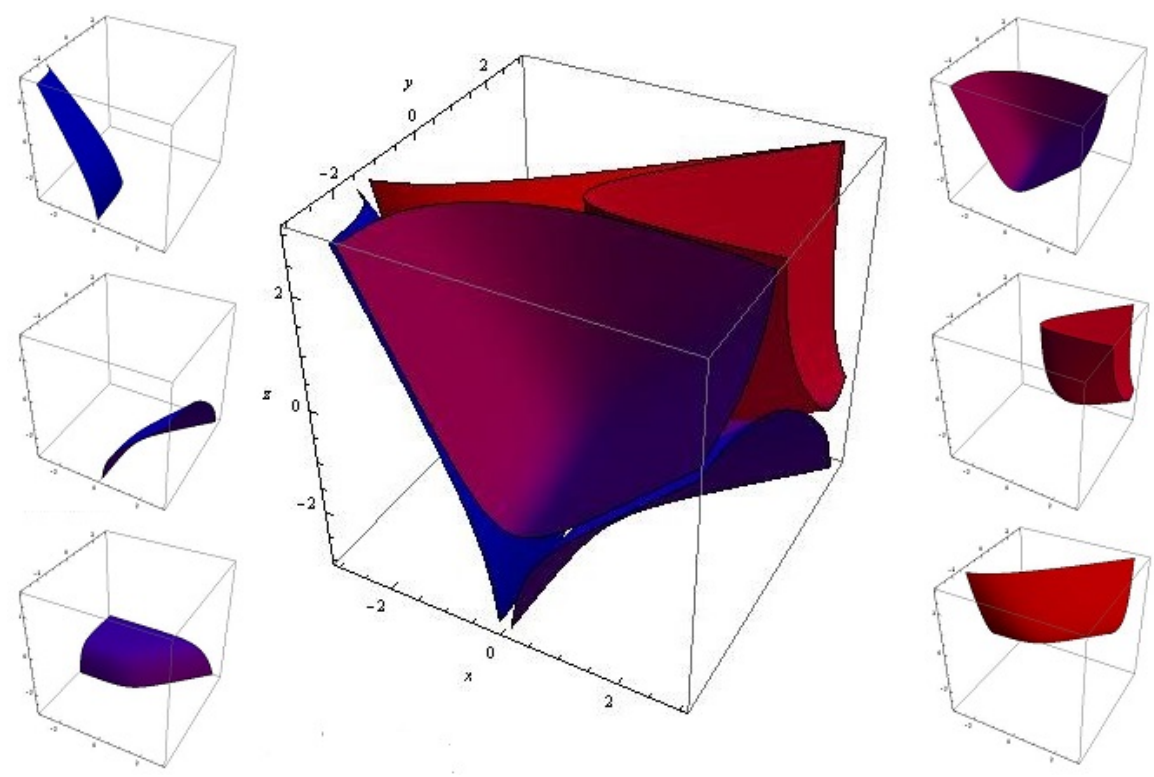

Figure 5. 1 -stratum with $f_{1} \equiv 0, f_{2} \equiv 3 x, f_{3} \equiv 3 y, f_{4} \equiv 2 x+z+\log 6, f_{5} \equiv$ $2 x+y+z+\log 11, f_{6} \equiv 3 y+z+\log 4$.

\section{Higher strata}

Higher strata $\mathcal{Z}_{\text {sing, }, k}$ have rather complicated structure. For instance, for $N=6$, $n=2$ and functions $f_{\alpha}(x, y)$ given, as in figure 3 , by $f_{1}(x, y) \equiv 0, f_{2}(x, y) \equiv 3 x$, $f_{3}(x, y) \equiv 3 y, f_{4}(x, y) \equiv x+y+\ln 6, f_{5}(x, y) \equiv 2 x+y+\ln 11, f_{6}(x, y) \equiv x+3 y+\ln 4$, 
the stratum $\mathcal{Z}_{\text {sing, } 2}$ is the set of 15 curves defined by $\frac{N(N-1)}{2}=15$ equations

$$
\mathcal{Z}_{2}(\{\alpha, \beta\} ; x, y):=\sum_{\gamma=1}^{6} g_{\{\alpha \beta\}(\gamma)} e^{f_{\gamma}(x, y)}, \quad \alpha \neq \beta, \alpha, \beta=1, \ldots, 6
$$

with $g_{\{\alpha \beta\}(\gamma)}=-\delta_{\alpha \gamma}-\delta_{\beta \gamma}$ at $\gamma \in\{\alpha, \beta\}, g_{\{\alpha \beta\}(\gamma)}=1$ at $\gamma \neq \alpha, \beta$. The stratum $\mathcal{Z}_{\text {sing, } 3}$ is the set of 20 curves defined by $\left(\begin{array}{c}N \\ k\end{array}\right)=20$ equations

$$
\mathcal{Z}_{3}(\{\alpha, \beta, \gamma\} ; x, y):=\sum_{\eta=1}^{6} g_{\{\alpha \beta \gamma\}(\eta)} e^{f_{\eta}(x, y)}, \quad \alpha \neq \beta \neq \gamma \neq \alpha, \alpha, \beta, \gamma=1, \ldots, N
$$

with $g_{\{\alpha \beta \gamma\}(\eta)}=-\delta_{\alpha \eta}-\delta_{\beta \eta}-\delta_{\gamma \eta}$ at $\eta \in\{\alpha, \beta, \gamma\}, g_{\{\alpha \beta \gamma\}(\eta)}=1$ at $\eta \neq \alpha, \beta, \gamma$. These sets of curves are presented in figure 1(a) and 1(b) respectively.

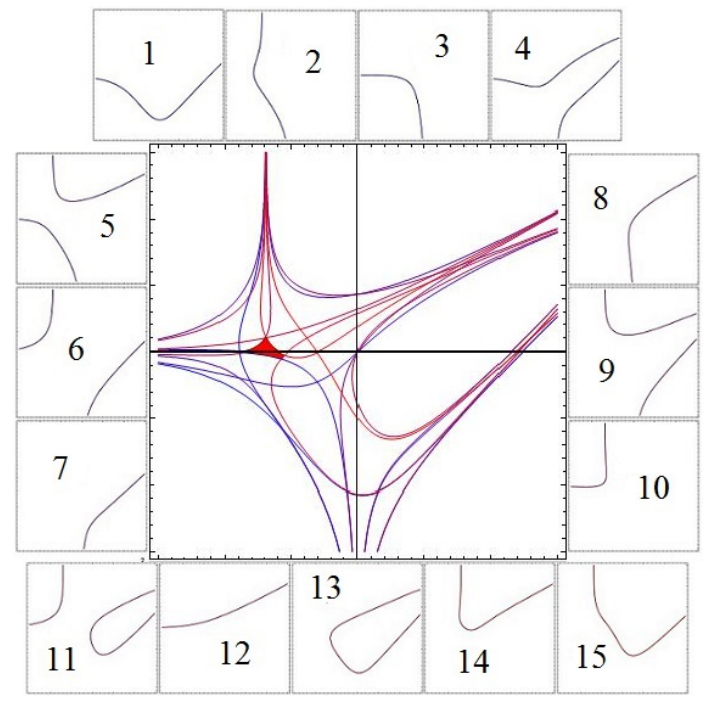

(a) 2-stratum.

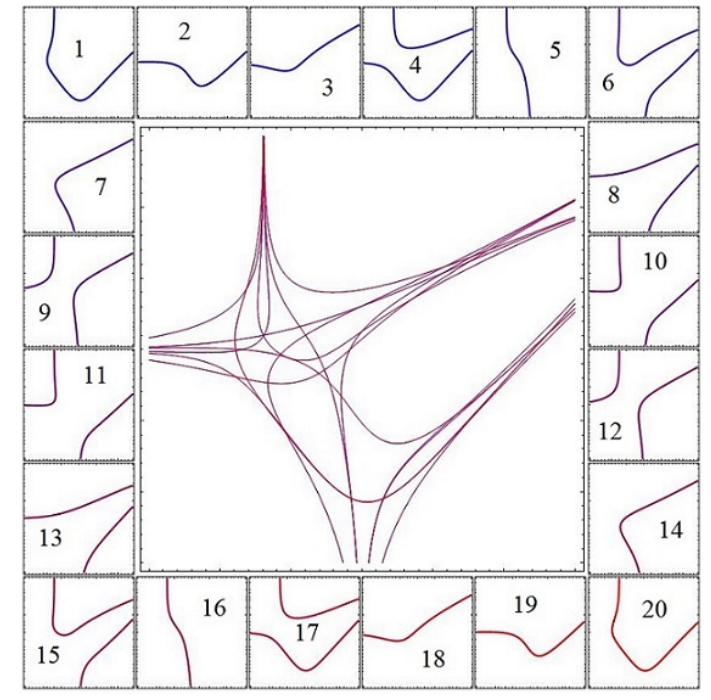

(b)3-stratum.

Figure 6. Higher strata with $f_{1} \equiv 0, f_{2} \equiv 3 x, f_{3} \equiv 3 y, f_{4} \equiv x+y+\ln 6, f_{5} \equiv 2 x+y+\ln 11$, $f_{6} \equiv x+3 y+\ln 4$. For each choice of 2 - and 3 - subsets, listed in lexicographical order, corresponding locus is shown. Components in $\mathcal{Z}_{\text {sing,3 } 3}$ are listed twice: curve $\alpha$ coincides with curve $21-\alpha, \alpha=1, \ldots, 10$, since they correspond to the same partition.

Due to the involution $P: g_{\alpha} \mapsto-g_{\alpha}$ the strata $\mathcal{Z}_{\text {sing, } 4}$ and $\mathcal{Z}_{\text {sing, } 5}$ coincide with 
strata $\mathcal{Z}_{\text {sing, }, 2}$ and $\mathcal{Z}_{\text {sing, } 1}$, respectively. The stratum $\mathcal{Z}_{\text {sing }, 3}$ is stable under involution, $P\left(\mathcal{Z}_{\text {sing }, 3}\right)=\mathcal{Z}_{\text {sing,3 }}$ and at most half of conditions in (3.2) are independent.

In the case $N=6$ and $n=3$ and with the same functions $f_{\alpha}$ as in figure 5 , i.e. $f_{1} \equiv 0, f_{2} \equiv 3 x, f_{3} \equiv 3 y, f_{4} \equiv 2 x+z+\log 6, f_{5} \equiv 2 x+y+z+\log 11$, $f_{6} \equiv 3 y+z+\log 4$, the strata $\mathcal{Z}_{\text {sing, } 2}$ and $\mathcal{Z}_{\text {sing }, 3}$ are given in figure 7 .

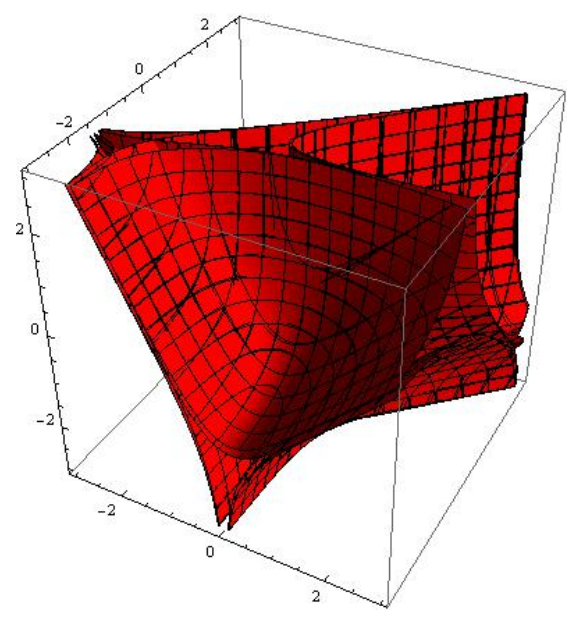

(a)2-stratum.

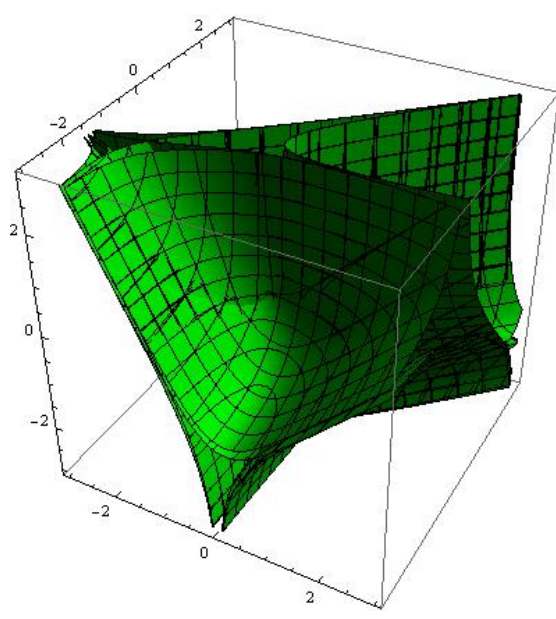

(b)3-stratum.

Figure 7. Examples of higher strata with $f_{1} \equiv 0, f_{2} \equiv 3 x, f_{3} \equiv 3 y, f_{4} \equiv 2 x+z+\log 6$, $f_{5} \equiv 2 x+y+z+\log 11, f_{6} \equiv 3 y+z+\log 4$.

In order to describe general properties of higher singular strata let us introduce some notation. We will denote by $\left(\mathcal{I}_{1}, \mathcal{I}_{2}\right)$ an ordered 2-partition of the set $[N]:=$ $\{1, \ldots, N\}$, i.e. the pair of two subsets $\mathcal{I}_{1}, \mathcal{I}_{2} \subseteq[N]$ such that $\mathcal{I}_{1} \cup \mathcal{I}_{2}=[N]$ and $\mathcal{I}_{1} \cap \mathcal{I}_{2}=\emptyset$. Then for each ordered 2-partition $(\mathcal{I},[N] \backslash \mathcal{I})$ we define the function

$$
\mathcal{Z}_{k}(\mathcal{I} ; \boldsymbol{x}):=-\sum_{\alpha \in \mathcal{I}_{1}} e^{f_{\alpha}(\boldsymbol{x})}+\sum_{\beta \in \mathcal{I}_{2}} e^{f_{\beta}(\boldsymbol{x})}
$$

and the corresponding zero locus as

$$
\mathcal{Z}_{\text {sing }, k}(\mathcal{I}):=\left\{\boldsymbol{x}: \mathcal{Z}_{k}(\mathcal{I} ; \boldsymbol{x})=0\right\}
$$

In the following we will ofter write $\mathcal{Z}_{k}(\mathcal{I})$ instead of $\mathcal{Z}_{k}(\mathcal{I} ; \boldsymbol{x})$ for notational convenience. 
Since $\mathcal{Z}_{N-k}\left(\mathcal{I}_{2}, \mathcal{I}_{1}\right)=-\mathcal{Z}_{k}\left(\mathcal{I}_{1}, \mathcal{I}_{2}\right)$ zero loci of $\mathcal{Z}_{k}\left(\mathcal{I}_{1}, \mathcal{I}_{2}\right)$ and $\mathcal{Z}_{N-k}\left(\mathcal{I}_{2}, \mathcal{I}_{1}\right)$ coincide. To avoid such redundancy we will focus on unordered partitions $\left\{\mathcal{I}_{1}, \mathcal{I}_{2}\right\}$ and we will assume in what follows for each partition $\left(\mathcal{I}_{1}, \mathcal{I}_{2}\right)$ the cardinality $\# \mathcal{I}_{1}$ of the first subset $\mathcal{I}_{1}$ is smaller than that of $\mathcal{I}_{2}$. Since $\mathcal{I}_{1} \cup \mathcal{I}_{2}=[N]$ and $\mathcal{I}_{1} \cap \mathcal{I}_{2}=\emptyset$ one has $0 \leq \# \mathcal{I}_{1} \leq\left\lfloor\frac{N}{2}\right\rfloor$.

Further for each subset $\mathcal{I}$ of $[N]$ of cardinality $k$ one has the equation

$$
\mathcal{Z}_{k}(\mathcal{I} ; \boldsymbol{x})=0
$$

which defines the hypersurface (3.4) in $\mathbb{R}^{n}$. Union of all such hypersurfaces with $\# \mathcal{I}=k$ is the stratum $\mathcal{Z}_{\text {sing, } k}$. Denoting the set of all subsets $\mathcal{I}$ of $[N]$ with $\# \mathcal{I}=k$ as $\mathcal{P}_{k}[N]$ one, hence, has

$$
\mathcal{Z}_{\text {sing }, k}=\bigcup_{\mathcal{I}_{\mathcal{P}}[N]} \mathcal{Z}_{\text {sing }, k}(\mathcal{I})
$$

Note also that

$$
\mathcal{Z}_{k}(\mathcal{I} ; \boldsymbol{x})=0 \Leftrightarrow 2 \cdot \sum_{\alpha \in \mathcal{I}} e^{f_{\alpha}(\boldsymbol{x})}=\mathcal{Z}_{0}(\boldsymbol{x})
$$

and

$$
\mathcal{Z}_{k}(\boldsymbol{x}):=\sum_{\mathcal{I} \in \mathcal{P}_{k}[N]} e^{f_{\mathcal{I}}(\boldsymbol{x})}=\left(\begin{array}{c}
N-1 \\
k-1
\end{array}\right) \cdot \mathcal{Z}_{0}(\boldsymbol{x})
$$

where $\mathcal{Z}_{0}(\boldsymbol{x})=\sum_{\alpha=1}^{N} e^{f_{\alpha}(\boldsymbol{x})}$ and we denote

$$
e^{f_{\mathcal{I}}(\boldsymbol{x})}:=\sum_{\alpha \in \mathcal{I}} e^{f_{\alpha}(\boldsymbol{x})}
$$

Figure 8 indicates that curves and hypersurfaces which compose higher strata may intersect in contrast to the stratum $\mathcal{Z}_{\text {sing, } 1}$. In general, one has

Proposition 1. Two hypersurfaces (3.5) belonging to the same stratum $\mathcal{Z}_{\text {sing,k }}$ and different $\mathcal{I}_{1}$ and $\mathcal{I}_{2}$ intersect at finite $\boldsymbol{x}$ only if $\mathcal{I}_{1} \cap \mathcal{I}_{2} \neq \emptyset$.

Proof. Consider two hypersurfaces $\mathcal{Z}_{\text {sing, } k}\left(\mathcal{I}_{1}\right)$ and $\mathcal{Z}_{\text {sing, } k}\left(\mathcal{I}_{2}\right)$ associated with two partitions of $[N]$ with $\# \mathcal{I}_{1}=\# \mathcal{I}_{2}=k$. Assume that they have a common point 


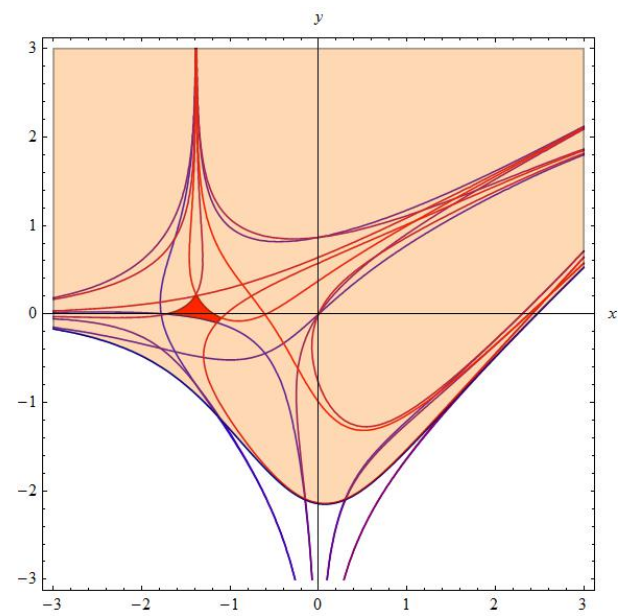

(a)Crossing of hypersurfaces can

happen for a higher stratum $\mathcal{Z}_{\text {sing, } 2}$.

Not all hypersurfaces lie in the same

halfspace defined by a certain

hypersurface, as it is for the orange

region defined by $\mathcal{Z}_{2}(\{1,2\})>0$. Red

region is the set where $\mathcal{Z}_{2}(\mathcal{I})>0$ for

all $\mathcal{I}$ with $\# \mathcal{I}=2$.

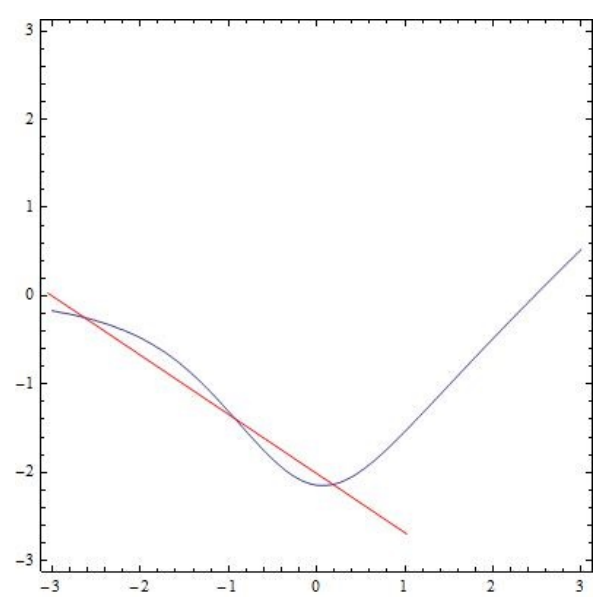

(b)Hypersurface defined by $\mathcal{Z}_{2}(\{1,2\} ; \boldsymbol{x})=0$. The red line shows that both regions of the plane bounded by this component are non-convex.

Figure 8. Comments on higher stratum $\mathcal{Z}_{\text {sing, } 2}$ with $f_{1} \equiv 0, f_{2} \equiv 3 x, f_{3} \equiv 3 y, f_{4} \equiv$ $x+y+\ln 6, f_{5} \equiv 2 x+y+\ln 11, f_{6} \equiv x+3 y+\ln 4$.

$\tilde{\boldsymbol{x}}$. Hence, one has

$$
\mathcal{Z}_{k}\left(\mathcal{I}_{1} ; \tilde{\boldsymbol{x}}\right)+\mathcal{Z}_{k}\left(\mathcal{I}_{2} ; \tilde{\boldsymbol{x}}\right)=2 \cdot \sum_{\alpha \in[N] \backslash\left(\mathcal{I}_{1} \cup \mathcal{I}_{2}\right)} e^{f_{\alpha}(\tilde{\boldsymbol{x}})}-2 \cdot \sum_{\beta \in \mathcal{I}_{1} \cap \mathcal{I}_{2}} e^{f_{\beta}(\tilde{\boldsymbol{x}})}=0 .
$$

If $\mathcal{I}_{1} \cup \mathcal{I}_{2} \neq[N]$ then this equation may have real solutions only if the second term is different from zero, i.e. $\mathcal{I}_{1} \cap \mathcal{I}_{2} \neq \emptyset$. If $\mathcal{I}_{1} \cup \mathcal{I}_{2}=[N]$ then $2 k \geq N$, but $k \leq \frac{N}{2}$ so $k=\frac{N}{2}$; then $\mathcal{I}_{1}$ and $\mathcal{I}_{2}$ are complementary sets and they define the same equation $\mathcal{Z}_{k}\left(\mathcal{I}_{1}, \mathcal{I}_{2}\right)=0$. 
Maximum number of intersections in stratum $\mathcal{Z}_{\text {sing, }, k}$ is bounded by one-half of the number of different pairs $\mathcal{I}_{1}$ and $\mathcal{I}_{2}$ with $\# \mathcal{I}_{1}=\# \mathcal{I}_{2}=k \leq\left\lfloor\frac{N}{2}\right\rfloor$ such that $\mathcal{I}_{1} \cap \mathcal{I}_{2} \neq \emptyset$. There are $\left(\begin{array}{l}N \\ k\end{array}\right)$ different partitions $(\mathcal{I},[N] \backslash \mathcal{I})$ of $[N]$ with cardinality $\# \mathcal{I}=k$ and for each such $\mathcal{I}$ there are $\left(\begin{array}{l}N \\ k\end{array}\right)-1-\left(\begin{array}{c}N-k \\ k\end{array}\right)$ partitions $(\mathcal{J},[N] \backslash \mathcal{J})$, $\mathcal{J} \neq \mathcal{I}$ and $\mathcal{I} \cap \mathcal{J} \neq \emptyset$. Then the number of intersections in the stratum $\mathcal{Z}_{\text {sing, } k}$ is bounded from above by $\frac{1}{2} \cdot\left(\begin{array}{l}N \\ k\end{array}\right) \cdot\left[\left(\begin{array}{l}N \\ k\end{array}\right)-1-\left(\begin{array}{c}N-k \\ k\end{array}\right)\right]$. It is not always a strict bound since some of these intersections might be unreachable. For example, at $k=2$ one has $\#\left(\mathcal{I}_{1} \cap \mathcal{I}_{2}\right)=1$ and, hence, $\mathcal{I}_{1}=\left\{\alpha, \gamma_{1}\right\}$ and $\mathcal{I}_{2}=\left\{\alpha, \gamma_{2}\right\}$ for some $\alpha \neq \gamma_{1} \neq \gamma_{2} \neq \alpha$. Then, if $\overline{\boldsymbol{x}}$ is in the intersection $\mathcal{Z}_{\text {sing, },}\left(\mathcal{I}_{1}\right) \cap \mathcal{Z}_{\text {sing, } 2}\left(\mathcal{I}_{2}\right)$ one has $e^{f_{\gamma_{1}}(\overline{\boldsymbol{x}})}-e^{f_{\gamma_{2}}(\overline{\boldsymbol{x}})}=-e^{f_{\alpha}(\overline{\boldsymbol{x}})}+\sum_{\beta \neq \alpha, \gamma_{1}, \gamma_{2}} e^{f_{\beta}(\overline{\boldsymbol{x}})}=e^{f_{\gamma_{2}}(\overline{\boldsymbol{x}})}-e^{f_{\gamma_{1}}(\overline{\boldsymbol{x}})}$, thus $f_{\gamma_{1}}(\overline{\boldsymbol{x}})=f_{\gamma_{2}}(\overline{\boldsymbol{x}})$ and $e^{f_{\alpha}(\overline{\boldsymbol{x}})}=\sum_{\beta \neq \alpha, \gamma_{1}, \gamma_{2}} e^{f_{\beta}(\overline{\boldsymbol{x}})}$. This last equation not always has real solutions for general choice of functions.

In general, intersections of hypersurfaces (3.5) for the stratum $\mathcal{Z}_{\text {sing, } k}$ are not necessarily transversal. Moreover, the assumption of real-valued functions opens up the way to reductions. For example, take two functions $g(\boldsymbol{x})$ and $h(\boldsymbol{x})$ and consider $f_{1}(\boldsymbol{x}) \equiv 4 \cdot g(\boldsymbol{x}), f_{2}(\boldsymbol{x}) \equiv 4 \cdot h(\boldsymbol{x}), f_{3}(\boldsymbol{x}) \equiv \ln 6+2 \cdot g(\boldsymbol{x})+2 \cdot h(\boldsymbol{x})$, $f_{4}(\boldsymbol{x})=\ln 4+3 \cdot g(\boldsymbol{x})+h(\boldsymbol{x}), f_{5}(\boldsymbol{x})=\ln 4+g(\boldsymbol{x})+3 \cdot h(\boldsymbol{x})$. Then $\mathcal{Z}_{2}(\{4,5\} ; \boldsymbol{x})=$ $\left(e^{2 g(\boldsymbol{x})}+e^{2 h(\boldsymbol{x})}-e^{\ln 2+g(\boldsymbol{x})+h(\boldsymbol{x})}\right)^{2} \geq 0$ and it vanishes if and only if $e^{2 g(\boldsymbol{x})}+e^{2 h(\boldsymbol{x})}=$ $e^{\ln 2+g(\boldsymbol{x})+h(\boldsymbol{x})}$. From Arithmetic-Geometric inequality, this is equivalent to the algebraic constraint $g(\boldsymbol{x})=h(\boldsymbol{x})$. Occurrence of such non-transversal crossings or reductions is a particular case and influences the investigation on equilibrium and non-equilibrium regions. We assume hereafter that pairwise intersections between hypersurfaces defined by $\mathcal{Z}_{k}(\mathcal{I})=0$ or $f_{\alpha}(\boldsymbol{x})-f_{\beta}(\boldsymbol{x})=0,1 \leq \alpha<\beta \leq N$ are transversal. 


\section{Higher strata. General properties}

All hypersurfaces (3.4) which compose the stratum $\mathcal{Z}_{\text {sing, }, k}$ divide $\mathbb{R}^{n}$ in a number of regions which we will call domains. Then, let us denote the subdomain in $\mathbb{R}^{n}$ where all functions $\mathcal{Z}_{k}(\mathcal{I})>0, \mathcal{I} \in \mathcal{P}_{k}[N]$, as $\mathcal{D}_{k+}$. For example, blue regions in figures 1,2 and 3 represent $\mathcal{D}_{1+}$ and the red region in $8(\mathrm{a})$ represents $\mathcal{D}_{2+}$. The domain $\mathbb{R}^{n} \backslash \mathcal{D}_{k+}$ is divided by hypersurfaces $\left\{\mathcal{Z}_{k}(\mathcal{I})=0\right\}$ into subdomains where some of functions $\mathcal{Z}_{k}(\mathcal{I})$ are positive and others are negative. Let us denote $\mathcal{D}_{k-}$ as the domain where the number of negative functions $\mathcal{Z}_{k}(\mathcal{J})$ with $\mathcal{J} \in$ $\mathcal{P}_{k}[N]$ is maximal. The hypersurfaces of the $k$-stratum are confined in certain domain which we will refer as zeros confinement domain $Z C D_{k}:=\mathbb{R}^{n} \backslash\left(\mathcal{D}_{k+} \cup \mathcal{D}_{k-}\right)$. The domain $Z C D_{k}$ is a sort of intermittent shell which separate the domains $\mathcal{D}_{k+}$ and $\mathcal{D}_{k-}$ and the boundary of $\mathcal{D}_{k+} \cup Z C D_{k}$ will be referred as the extremal points of hypersurfaces composing $\mathcal{Z}_{\text {sing, } k}$. For the first stratum $V_{1}$ defined in (2.2) the domain $Z C D_{1}$ generically has dimension $n-1$ and consists of hypersurfaces $\left\{\boldsymbol{x}: \mathcal{Z}_{1}(\{\alpha\} ; \boldsymbol{x})=0\right\}, \alpha=1, \ldots, N$ themselves. For higher $V_{k}, k \geq 2$, the domain $Z C D_{k}$ has generically dimension $n$ and its boundary is tipically formed by pieces of different hypersurfaces belonging to $\mathcal{Z}_{\text {sing, }, k}$.

Since at $\mathcal{D}_{k+}$ the partition function $\left.\mathcal{Z}_{k} \equiv \mathcal{Z}\right|_{V_{k}}>0$ it is natural to refer to the domain $\mathcal{D}_{k+}$ as stability (equilibrium) domain. It is surrounded by the domain $Z C D_{k}$ with rather complicated singularity structure. The domain $\mathcal{D}_{k-}$ is an ambient instability domain.

These domains for different strata exhibit a simple inclusion chain.

Proposition 2. Let $1 \leq k<\hat{k} \leq\left\lfloor\frac{N}{2}\right\rfloor$ then $\mathcal{D}_{\hat{k}+} \subseteq \mathcal{D}_{k+}$.

Proof. Through the proof is an immediate consequence of the definition of $\mathcal{D}_{k+}$, we present it here for completeness. Let $\hat{k}>k$ and $\mathcal{I}_{k} \subset \mathcal{I}_{\hat{k}}$ be two subsets of $[N]$ 
such that $\# \mathcal{I}_{k}=k$ and $\# \mathcal{I}_{\hat{k}}=\hat{k}$. Then for any $\boldsymbol{x} \in \mathbb{R}^{n}$ one has the identity

$$
\mathcal{Z}_{k}\left(\mathcal{I}_{k} ; \boldsymbol{x}\right)-\mathcal{Z}_{\hat{k}}\left(\mathcal{I}_{\hat{k}} ; \boldsymbol{x}\right)=2 \cdot \sum_{\alpha \in \mathcal{I}_{k+1} \backslash \mathcal{I}_{k}} e^{f_{\alpha}(\boldsymbol{x})}>0 .
$$

In the domain $\mathcal{D}_{\hat{k}+}$ one has $\mathcal{Z}_{\hat{k}}\left(\mathcal{I}_{\hat{k}} ; \boldsymbol{x}\right)>0$ for all subsets $\mathcal{I}_{k+1}$. Inequality (4.1) implies that all $\mathcal{Z}_{k}\left(\mathcal{I}_{k} ; \boldsymbol{x}\right)>0$ in $\mathcal{D}_{\hat{k}+}$ too. So $\mathcal{D}_{\hat{k}+} \subseteq \mathcal{D}_{k+}$.

Thus, one has the inclusion chain

$$
\mathcal{D}_{0+}=\mathbb{R}^{n} \supseteq \mathcal{D}_{1+} \supseteq \mathcal{D}_{2+} \supseteq \cdots \supseteq \mathcal{D}_{\left\lfloor\frac{N}{2}\right\rfloor+}
$$

An example with $N=7$ and $f_{\alpha}(x, y)$ as in (2.9) is given in figure 9, where the domain $\mathcal{D}_{1+}$ is shown in blue color, $\mathcal{D}_{2+}$ in red and $\mathcal{D}_{3+}$ in green.

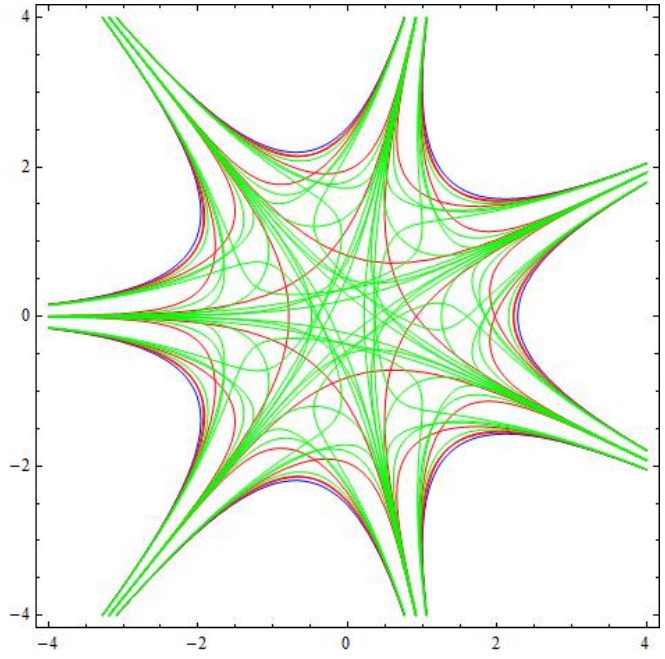

(a) Singular sectors $\mathcal{Z}_{\text {sing }, 1}$ (blue), $\mathcal{Z}_{\text {sing }, 2}$ (red) and $\mathcal{Z}_{\text {sing,3 }}$ (green).

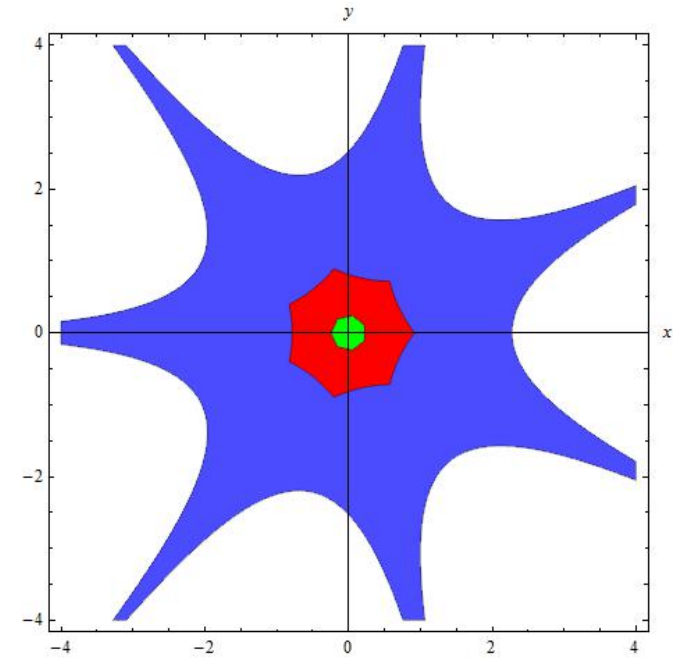

(b)Domains $\mathcal{D}_{3+} \subseteq \mathcal{D}_{2+} \subseteq D_{1+}$.

Figure 9. Inclusion chain for equilibrium domains in the case $f_{\alpha} \equiv \cos \left(\frac{2 \pi(\alpha-1)}{7}\right) x+$ $\sin \left(\frac{2 \pi(\alpha-1)}{7}\right) y, \alpha=1, \ldots, 7$.

Furthermore, one also has 
Proposition 3. Let us take $1 \leq k<\hat{k} \leq\left\lfloor\frac{N}{2}\right\rfloor$. Then, there exists a dense subset of $\mathcal{Z}_{\text {sing, }, \hat{k}}$ such that each ray $(\vec{r})_{i}=x_{0, i}+t \cdot e_{i}$ originated from this set, $\boldsymbol{e} \in \mathbb{R}^{n}$, intersects $\mathcal{Z}_{\text {sing }, k}$.

In other words, if $\left\{f_{\alpha}(\boldsymbol{x}): \alpha \in[N]\right\}$ are pairwise different linear functions, then $\mathcal{Z}_{\text {sing }, \hat{k}}$ lies inside a region of $\mathbb{R}^{n}$ delimited by some components of $\mathcal{Z}_{\text {sing }, k}$, $1 \leq k<\hat{k} \leq\left\lfloor\frac{N}{2}\right\rfloor$.

Proof. The proof is based on the following

Lemma 1. Consider any ray $\vec{r}$ with base point $\boldsymbol{x}$, slopes $\boldsymbol{e}:=\left(e_{1}, \ldots, e_{n}\right)$ and parametrization $\vec{r}(t):=\boldsymbol{x}+t \cdot \boldsymbol{e}, t \geq 0$. If none of functions $f_{\alpha}(\vec{r}(t))-f_{\beta}(\vec{r}(t))$ vanishes identically, $\alpha \neq \beta$, then there exists $t_{0} \in \mathbb{R}_{+}$such that $\vec{r}(t)$ belongs to a region in $\mathbb{R}^{n}$ where there is only one dominant function $f_{\alpha}, \alpha \in[N]$, at $t \geq t_{0}$, i.e. $\#\left\{\alpha \in[N]: \forall \beta \in[N], f_{\alpha}(\vec{r}(t)) \geq f_{\beta}(\vec{r}(t))\right\}=1$.

Proof. Let us consider the $\frac{N(N-1)}{2}$ functions

$$
d_{\alpha \beta}(t):=f_{\alpha}(\vec{r}(t))-f_{\beta}(\vec{r}(t)) \quad 1 \leq \alpha<\beta \leq N .
$$

Since none of these linear functions vanishes identically, each of them has a finite number of roots, so the set of points

$$
\Omega:=\bigcup_{1 \leq \alpha<\beta \leq N}\left\{t: d_{\alpha \beta}(t)=0\right\}
$$

is finite. Thus, for $t_{0}>\max (\Omega)$, all $d_{\alpha \beta}\left(t_{0}\right)$ will be definitely different from zero, hence all $\left\{f_{\alpha}\left(\vec{r}\left(t_{0}\right)\right): \alpha \in[N]\right\}$ are pairwise different. In particular, there will be one and only one $\alpha_{0} \in[N]$ such that $f_{\alpha_{0}}\left(\vec{r}\left(t_{0}\right)\right)>f_{\alpha}\left(\vec{r}\left(t_{0}\right)\right)$ for all $\alpha \neq \alpha_{0}$. So $f_{\alpha_{0}}(\vec{r}(t))-f_{\alpha}(\vec{r}(t))>0$ at $t>t_{0}$ too, since a change of sign would imply an additional zero of $d_{\alpha_{0} \alpha}$ by continuity.

Now, let $(\mathcal{J},[N] \backslash \mathcal{J})$ be any partition of $[N]$ such that $\# \mathcal{J}=\hat{k} \leq\left\lfloor\frac{N}{2}\right\rfloor$ and $\vec{r}(t):=\left(x_{i}+t \cdot e_{i}\right), t \geq 0$ be a ray with base point $\boldsymbol{x} \in \mathcal{Z}_{\text {sing, }, \hat{k}}$. If $d_{\alpha \beta}(t)$ in $(4.3)$ 
does not vanish identically for all $\alpha<\beta$, then lemma 1 implies that there exist $t_{0}>0$ and $\alpha_{0} \in[N]$ such that one has $d_{\beta \alpha_{0}}(t)<0$ at $t \geq t_{0}$ and $\beta \neq \alpha_{0}$. In particular, $d_{\beta \alpha_{0}}$ are linear functions of $t$ and they are negative at $t \geq t_{0}$. Hence one has

$$
\lim _{t \rightarrow+\infty} f_{\beta}(\vec{r}(t))-f_{\alpha_{0}}(\vec{r}(t))=-\infty, \quad \beta \neq \alpha_{0} .
$$

So there exists $t_{1}>t_{0}$ such that $\sum_{\beta \neq \alpha_{0}} e^{f_{\beta}(\vec{r}(t))-f_{\alpha_{0}}(\vec{r}(t))}<1$ at $t>t_{1}$. The index $\alpha_{0}$ belongs to only one subset $\mathcal{J}$ or $[N] \backslash \mathcal{J}$, call it $\mathcal{J}\left(\alpha_{0}\right)$ : from $k<\hat{k} \leq N-\hat{k}$ it follows that $k<\# \mathcal{J}\left(\alpha_{0}\right)$ and one can always choose a subset $\mathcal{I} \subset \mathcal{J}\left(\alpha_{0}\right)$ with $k$ elements such that $\alpha_{0} \in \mathcal{I}$. One has $\left.\vec{r}(t)\right|_{t=0}=\boldsymbol{x}$ so $\sum_{\alpha \in \mathcal{I}} e^{f_{\alpha}(\boldsymbol{x})}<$ $\sum_{\alpha \in \mathcal{J}\left(\alpha_{0}\right)} e^{f_{\alpha}(\boldsymbol{x})}=\sum_{\beta \in[N] \backslash \mathcal{J}\left(\alpha_{0}\right)} e^{f_{\beta}(\boldsymbol{x})}<\sum_{\beta \in[N] \backslash \mathcal{I}} e^{f_{\beta}(\boldsymbol{x})}$. On the other hand, at $t=t_{1}$ one has $\sum_{\alpha \in \mathcal{I}} e^{f_{\alpha}\left(\vec{r}\left(t_{1}\right)\right)}>e^{f_{\alpha_{0}}\left(\vec{r}\left(t_{1}\right)\right)}>\sum_{\beta \neq \alpha_{0}} e^{f_{\beta}\left(\vec{r}\left(t_{1}\right)\right)}>\sum_{\beta \in[N] \backslash \mathcal{I}} e^{f_{\beta}\left(\vec{r}\left(t_{1}\right)\right)}$. Then, there exists a point $0<\bar{t}<t_{1}$ such that $\sum_{\alpha \in \mathcal{I}} e^{f_{\alpha}(\vec{r}(\bar{t}))}=\sum_{\beta \in[N] \backslash \mathcal{I}} e^{f_{\beta}(\vec{r}(\bar{t}))}$ by continuity. Thus $\vec{r}(\bar{t})$ is in the set $\mathcal{Z}_{\text {sing, }, k}(\mathcal{I}) \subseteq \mathcal{Z}_{\text {sing, }, k}$. If instead $d_{\alpha_{0} \beta_{0}}(t) \equiv 0$ for some $\alpha_{0} \neq \beta_{0}$ at the point $\left(\boldsymbol{x}_{\mathbf{0}} ; \boldsymbol{e}\right)$, then $f_{\alpha_{0}}\left(\boldsymbol{x}_{\mathbf{0}}\right)-f_{\beta_{0}}\left(\boldsymbol{x}_{\mathbf{0}}\right)=d_{\alpha_{0} \beta_{0}}(0)=0$ and $\boldsymbol{x}_{\mathbf{0}} \in \mathcal{Z}_{\text {sing, }, k} \cap\left\{\boldsymbol{x} \in \mathbb{R}^{n}: f_{\alpha_{0}}(\boldsymbol{x})=f_{\beta_{0}}(\boldsymbol{x})\right\}$. From the generic hypothesis of transversal crossing, the complement of set of such points $\boldsymbol{x}_{\mathbf{0}}$ in $\mathcal{Z}_{\text {sing, } \hat{k}}$ is dense.

Some concrete examples are presented in figure 10.

Note that the rays that do not come out $Z C D_{k}$ are connected with locus of coincident dominant functions $f_{\alpha}(\boldsymbol{x})=f_{\beta}(\boldsymbol{x})=\max _{\gamma}\left\{f_{\gamma}(\boldsymbol{x})\right\}, \alpha \neq \beta$, see figure 11(b).

\section{Statistical amoebas vs. algebraic amoebas}

We will refer to the domains $\mathcal{A}_{k}:=\mathcal{D}_{k+} \cup Z C D_{k}$ described in previous section as the statistical $k$-amoebas. They, generically, are composed by the internal stable nuclei (domains $\mathcal{D}_{k+}$ ) and enveloping shells $Z C D_{k}$ which contain singular 


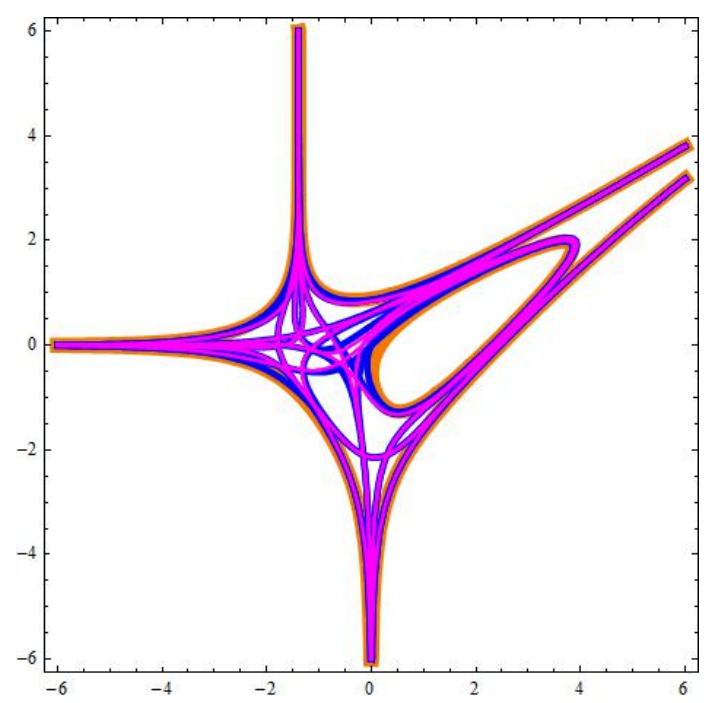

(a)Stratification of $\mathcal{Z}_{\text {sing, } 3}$ (magenta), $\mathcal{Z}_{\text {sing }, 2}$ (blue) and $\mathcal{Z}_{\text {sing }, 1}$ (orange) in case $f_{1} \equiv 0$,

$$
\begin{gathered}
f_{2} \equiv 3 x, f_{3} \equiv 3 y, f_{4} \equiv x+y+\ln 6 \\
f_{5} \equiv 2 x+y+\ln 11, f_{6} \equiv x+3 y+\ln 4
\end{gathered}
$$

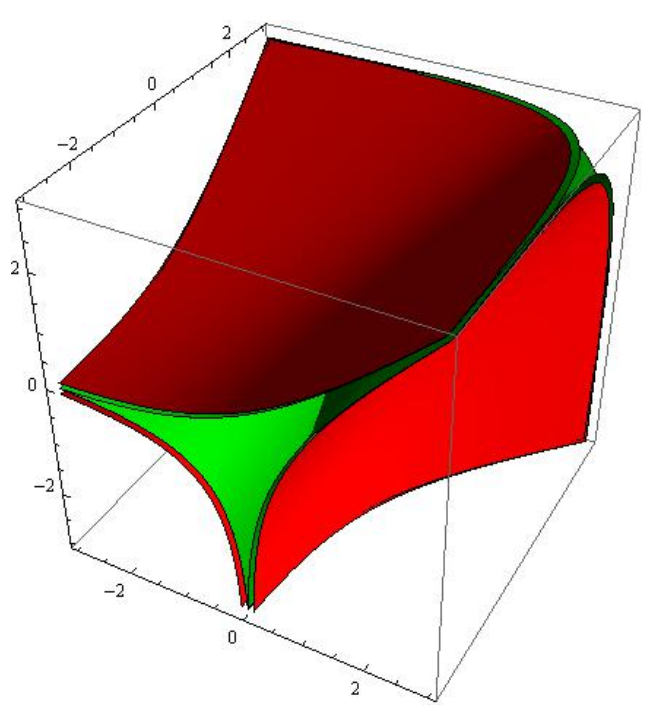

(b)Stratification of $\mathcal{Z}_{\text {sing,3 }}$ (green) and $\mathcal{Z}_{\text {sing }, 2}($ red) in $N=3$ super-ideal case.

Figure 10. Examples of stratifications.

hypersurfaces $\mathcal{Z}_{\text {sing,k }}(\mathcal{I})$ and subdomains with some number of positive and negative partition functions. The statistical amoeba $\mathcal{A}_{k}$ is surrounded by the domain $\mathcal{D}_{k-}$ of maximal instability (as we will demonstrate in next section).

For $k>\left\lfloor\frac{N}{2}\right\rfloor$ the domain $Z C D_{k}$ coincides with that of $Z C D_{N-k}$ while the domains $\mathcal{D}_{k-}$ and $\mathcal{D}_{k+}$ exchange their roles, namely $\mathcal{D}_{k-}:=\mathcal{D}_{(N-k)+}$ and $\mathcal{D}_{k+}:=$ $\mathcal{D}_{(N-k)-}$. With increasing $k$ the stability domain schrinks (proposition 2) while instable domain $\mathcal{D}_{k-}$ expands. At $k=N$ the whole space $\mathbb{R}^{n}$ is the domain of instability. It would be natural to refer to the domain $\mathcal{D}_{k-} \cup Z C D_{k}$ at $k>\left\lfloor\frac{N}{2}\right\rfloor$ as the statistical $k$-antiamoeba.

The name amoeba is borrowed from algebraic geometry. The amoeba of the 


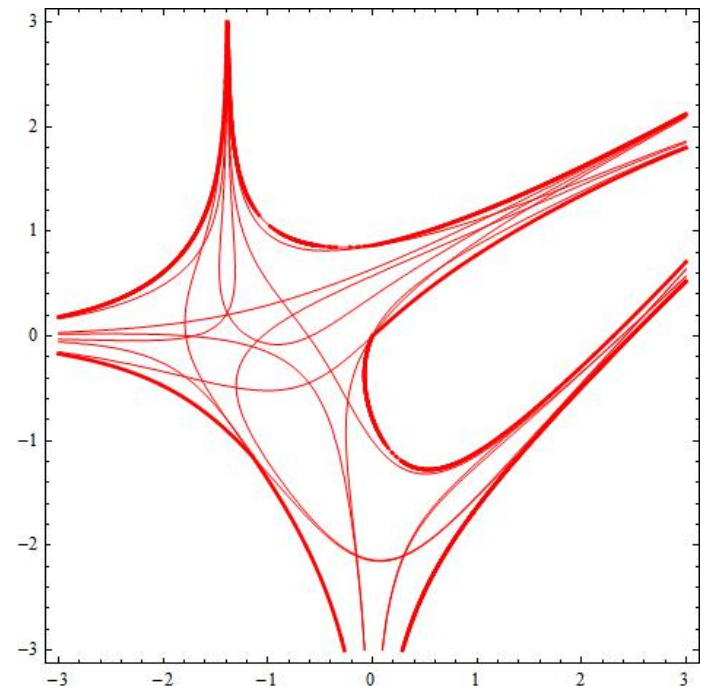

(a)2-stratum $\mathcal{Z}_{\text {sing, } 2}$; thick locus is the set of extremal points.

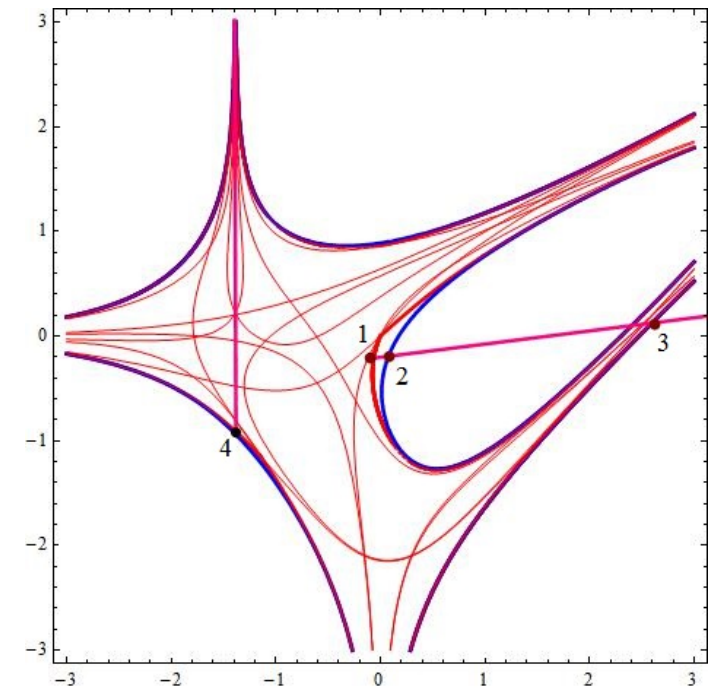

(b)Ray from 1, an extremal point of $\mathcal{Z}_{\text {sing, }, 2}$,

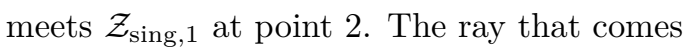
out from point 2 meets other strata, but the last one is $\mathcal{Z}_{\text {sing }, 1}$ itself. Ray from point $3 \in \mathcal{Z}_{\text {sing, } 1}$ does not meet other strata. Point 4 is in a particular position and never comes out $Z C D_{2}$.

Figure 11. Comments on relative positions of singular loci.

algebraic variety $\mathcal{V}_{n}$ given by the algebraic equation

$$
\sum_{m_{1}, \ldots, m_{n}} c_{m_{1}, \ldots, m_{n}} z_{1}^{m_{1}} z_{2}^{m_{2}} \cdots z_{n}^{m_{n}}=0
$$

with complex $z_{1}, \ldots, z_{n}$ and $c_{m_{1}, \ldots, m_{n}}$ is defined ${ }^{38}$ as the image of $\mathcal{V}_{n}$ under logarithmic map $\left(z_{1}, \ldots, z_{n}\right) \mapsto\left(\log \left|z_{1}\right|, \ldots, \log \left|z_{n}\right|\right)$. Amoebas of algebraic varieties and their properties have been intensively studied since their introduction by Gelfand, Kapranov and Zelevinsky (see e.g. ${ }^{31,39-44}$ ).

In the simplest esample of the complex plane given by the equation $1+z_{1}+z_{2}=0$ 
$\left(\right.$ see $\left.^{46}\right)$ the amoeba is defined by the triangle inequalities

$$
\begin{aligned}
& e^{x}+e^{y}>1, \\
& 1+e^{y}>e^{x}, \\
& 1+e^{x}>e^{y}
\end{aligned}
$$

and is presented in figure 1(a) (colored region). So in this case statistical and algebraic amoebas coincide. However, in general, it is not so. Indeed let us, firstly, rewrite equation (5.1) in the form

$$
\sum_{\alpha=1}^{N} \exp \left(b_{\alpha}+\sum_{i=1}^{n} a_{\alpha i} x_{i}+\mathrm{i}\left(\arg a_{\alpha}+\sum_{i=1}^{n} a_{\alpha i} \varphi_{i}\right)\right)=0
$$

where $x_{i}=\log \left|z_{i}\right|, \varphi_{i}=\arg z_{i}, a_{\alpha i}=e^{b_{\alpha i}+\mathrm{i} \arg a_{\alpha i}}$ and rows of $a_{\alpha i}$ are given by integers $m_{i}\left(a_{\alpha i}=m_{i}\right.$ for given monomial indexed by $\left.\alpha\right)$. Projection of the $2 n-2$ dimensional real hypersurface given by (5.3) onto the space $\mathbb{R}^{n}$ with coordinates $\left(x_{1}, \ldots, x_{n}\right)$ is the amoeba $\mathcal{A}$ of this hypersurface ${ }^{39-46}$.

On the other hand applying the triangle inequality to (5.3), one gets the set of inequalities

$$
-e^{f_{\alpha}(\boldsymbol{x})}+\sum_{\beta \neq \alpha} e^{f_{\beta}(\boldsymbol{x})}>0
$$

where $f_{\alpha}(\boldsymbol{x})=b_{\alpha}+\sum_{i=1}^{n} a_{\alpha i} x_{i}$. The domain in $\mathbb{R}^{n}$ defined by $N$ inequalities (5.4) is called approximated amoeba (non-lopsided set) $\mathcal{L} \mathcal{A}^{31}$. In general $\mathcal{L} \mathcal{A}$ does not coincide with the amoeba $\mathcal{A}$, namely $\mathcal{L} \mathcal{A} \supseteq \mathcal{A}$, but " $\mathcal{L} \mathcal{A}$ is a very good approximation for $\mathcal{A}^{\prime 31}$.

Comparing the set of inequalities (5.4) with our definition of the domain $\mathcal{D}_{1+}$, we can conclude that the statistical 1-amoeba with integer-valued elements $a_{\alpha i}$ and $f_{\alpha}(\boldsymbol{x}) \equiv b_{\alpha}+\sum_{i=1}^{n} a_{\alpha i} x_{i}$ in $(2.4)$ coincide with $\mathcal{L} \mathcal{A}$ amoeba for the hypersurface (5.3). The triangle inequalities reasoning becomes rather involved for partitions different from $\mathcal{I}_{1}=\{\alpha\}$ and $\mathcal{I}_{2}=[N] \backslash\{\alpha\}, \alpha=1, \ldots, N$. Anyway, equilibrium domains $\mathcal{D}_{k+}$ have a simple geometrical interpretation. First, one has the following well-known 
Lemma 2. If $\boldsymbol{x}$ is in the 1-equilibrium region $\mathcal{D}_{1+}$, then one can construct a polygonal closed path with $N$ sides of lengths $\left(e^{f_{\alpha}(\boldsymbol{x})}: \alpha \in[N]\right)$ in some order.

Proof. The base case $N=3$ is equivalent to triangle inequality. Then we assume that the assertion holds for all integers $k$ such that $N-1 \geq k \geq 3$ and will proceed by induction on $N$. One can fix $f_{1}(\boldsymbol{x}) \geq f_{2}(\boldsymbol{x}) \geq \cdots \geq f_{N}(\boldsymbol{x})$ without loss of generality and choose a number $\ell$ such that

$$
\max \left\{e^{f_{2}(\boldsymbol{x})}, e^{f_{1}(\boldsymbol{x})}-e^{f_{N}(\boldsymbol{x})}\right\} \leq \ell \leq \min \left\{e^{f_{1}(\boldsymbol{x})}, \sum_{\beta=2}^{N-1} e^{f_{\beta}(\boldsymbol{x})}\right\}
$$

Note that this definition is well-posed: $e^{f_{1}(\boldsymbol{x})}-e^{f_{N}(\boldsymbol{x})}<e^{f_{1}(\boldsymbol{x})}$ since $f_{N}(\boldsymbol{x})$ is real, $e^{f_{1}(\boldsymbol{x})}-e^{f_{N}(\boldsymbol{x})}<\sum_{\beta=2}^{N-1} e^{f_{\beta}(\boldsymbol{x})}$ since $\boldsymbol{x}$ belongs to the equilibrium region, $e^{f_{2}(\boldsymbol{x})} \leq e^{f_{1}(\boldsymbol{x})}$ by hypothesis and $e^{f_{2}(\boldsymbol{x})}<\sum_{\beta=2}^{N-1} e^{f_{\beta}(\boldsymbol{x})}$ since $N>3$. So there is at least one positive term. Let us consider

$$
\Lambda_{1}:=\left(e^{f_{1}(\boldsymbol{x})}, \ell, e^{f_{N}(\boldsymbol{x})}\right), \quad \Lambda_{2}:=\left(\ell, e^{f_{2}(\boldsymbol{x})}, \ldots, e^{f_{N-1}(\boldsymbol{x})}\right)
$$

One has $e^{f_{1}(\boldsymbol{x})}=e^{f_{1}(\boldsymbol{x})}-e^{f_{N}(\boldsymbol{x})}+e^{f_{N}(\boldsymbol{x})}<\ell+e^{f_{N}(\boldsymbol{x})}$ and $e^{f_{1}(\boldsymbol{x})}=\max \left\{e^{f_{1}(\boldsymbol{x})}, e^{f_{N}(\boldsymbol{x})}, \ell\right\}$ so $\Lambda_{1}$ is non-lopsided. One can see that $\ell=\max \Lambda_{2}$ and $\ell<\sum_{\beta=2}^{N-1} e^{f_{\beta}(x)}$ by construction. Then both $\Lambda_{1}$ and $\Lambda_{2}$ are non-lopsided and their cardinalities are 3 and $N-1$. Thus, by induction hypothesis there exists two polygonal closed paths $\mathcal{C}_{1}$ and $\mathcal{C}_{2}$ with sides $\left(e^{f_{1}(\boldsymbol{x})}, \ell, e^{f_{N}(\boldsymbol{x})}\right)$ and $\left(\ell, e^{f_{2}(\boldsymbol{x})}, \ldots, e^{f_{N-1}(\boldsymbol{x})}\right)$ in some order. Finally, one can join $\mathcal{C}_{1}$ and $\mathcal{C}_{2}$ along the side of length $\ell$ and get a closed polygonal path with $N$ sides of lengths $\left(e^{f_{\alpha}(x)}: \alpha \in[N]\right)$.

Stratification (4.2) can now be seen as a refinement of the triangle inequality property.

Proposition 4. The equilibrium domain $\mathcal{D}_{k+}$ relative to the $k$-amoeba is the set of all points $\boldsymbol{x}$ in $\mathbb{R}^{n}$ that satisfy the following condition: there exists a planar polygon 
with $g$ sides, for all $N-k+1 \leq g \leq N$, and with lengths of sides $\left(\ell_{1}, \ldots, \ell_{g}\right)$ equal to $\left(\sum_{\alpha \in \mathcal{I}_{1}} e^{f_{\alpha}(\boldsymbol{x})}, \ldots, \sum_{\alpha \in \mathcal{I}_{g}} e^{f_{\alpha}(\boldsymbol{x})}\right)$, where $\left\{\mathcal{I}_{1}, \ldots, \mathcal{I}_{g}\right\}$ is any partition of $[N]$ in $g$ disjoint non-empty subsets.

Proof. Let us suppose that $\boldsymbol{x} \in \mathcal{D}_{k+}$ and consider any $g$-partition $\left\{\mathcal{I}_{1}, \ldots, \mathcal{I}_{g}\right\}$ of $[N]$. Since all subsets in $\mathcal{I}_{1}, \ldots, \mathcal{I}_{g}$ are not empty, then $\# \mathcal{I}_{u} \geq 1$ for all $1 \leq u \leq g$. Thus

$$
\# \mathcal{I}_{u}=N-\sum_{w \neq u} \# \mathcal{I}_{w} \leq N-(g-1) \leq N-(N-k+1-1)=k, \quad 1 \leq u \leq g
$$

Assuming that $\boldsymbol{x} \in \mathcal{D}_{k+}$ one gets

$$
\sum_{\alpha \in \mathcal{I}_{u}} e^{f_{\alpha}(\boldsymbol{x})}<\sum_{\beta \notin \mathcal{I}_{u}} e^{f_{\beta}(\boldsymbol{x})}=\sum_{w \neq u} \sum_{\beta \in \mathcal{I}_{w}} e^{f_{\beta}(\boldsymbol{x})}, \quad 1 \leq u \leq g .
$$

By lemma 2, this is equivalent to the existence of a closed planar polygon with $g$ sides whose lengths are (in some order) $\sum_{\alpha \in \mathcal{I}_{1}} e^{f_{\alpha}(\boldsymbol{x})}, \ldots, \sum_{\alpha \in \mathcal{I}_{g}} e^{f_{\alpha}(\boldsymbol{x})}$.

Now assume that the existence hypothesis holds. In particular, it holds at $g=$ $N-k+1$ For any $\mathcal{I} \in \mathcal{P}_{k}[N]$ one can consider the $g$-partition $\left\{\left\{\beta_{1}\right\}, \ldots,\left\{\beta_{g-1}\right\}, \mathcal{I}\right\}$ where $[N] \backslash \mathcal{I}=:\left\{\beta_{1}, \ldots, \beta_{g-1}\right\}$. The hypothesis in such a case implies that $\sum_{\alpha \in \mathcal{I}} e^{f_{\alpha}(\boldsymbol{x})}<\sum_{u=1}^{g-1} e^{f_{\beta_{u}}(\boldsymbol{x})}=\sum_{\beta \notin \mathcal{I}} e^{f_{\beta}(\boldsymbol{x})}$. This means that $\mathcal{Z}_{k}(\mathcal{I} ; \boldsymbol{x})>0$ for all $\mathcal{I} \in$ $\mathcal{P}_{k}[N]$, that is $\boldsymbol{x} \in \mathcal{D}_{k+}$.

It seems that these higher amoebas, i.e. those defined by sets of inequalities of the type (5.4) with more than one minus sign were not discussed before in this context.

Some important features of algebraic amoebas are not preserved in the general $k$-amoeba case. For example, the extremal boundary of standard 1-amoebas is the set of points $\boldsymbol{x} \in \mathbb{R}^{n}$ such that $\boldsymbol{x} \in \mathcal{Z}_{\text {sing, } 1}(\{\alpha\})$ for certain $\alpha \in[N]$. All non-extremal points are partitioned in two sets defined by the sign of $\mathcal{Z}_{1}(\{\alpha\})$. As already noted, such partitions at different values of $\alpha$ are compatible, in the sense 
that if $\mathcal{Z}_{1}(\{\alpha\})<0$ then one knows that $\mathcal{Z}_{1}(\{\beta\})>0$ for all $\beta \neq \alpha$. Conversely, crossing points in $\mathcal{Z}_{\text {sing }, k}\left(\mathcal{I}_{1}\right) \cap \mathcal{Z}_{\text {sing }, k}\left(\mathcal{I}_{2}\right)$ between distinct components of the $k$ singular locus at $k \geq 2$ open the way for more sign combinations, see figure 8(a).

Furthermore, each connected component of the boundary of a standard algebraic amoeba bounds a certain convex (finite or infinite) region of the space (see e.g. ${ }^{38}$ ). This property does not hold in general for $k$-singular loci $\mathcal{Z}_{\text {sing, } k}(\mathcal{I})$, $\mathcal{I} \in \mathcal{P}_{k}[N]$. Such a case is pointed out in figure $8(\mathrm{~b})$.

Anyway, a generalization of these properties to $k$-amoebas can be done taking in account all $k$-singular loci $\mathcal{Z}_{\text {sing, } k}(\mathcal{I})$ simultaneously. Thus, in our real-valued approach higher statistical amoebas arise in a natural way.

We note also that the relations between 1-statistical amoebas and statistical physics have been discussed in ${ }^{47}$ and $^{32,34}$.

\section{Structure of ZCD domains}

Zeros confinement domain $Z C D_{k}$ for $k$-stratum separating the domains $\mathcal{D}_{k+}$ and $\mathcal{D}_{k-}$ has rather complicated structure in general. Here we will consider some of their simplest properties.

For the first stratum $k=1$ the $Z C D_{1}$ collapses into the set of hypersurfaces of zeros $\mathcal{Z}_{\text {sing, }, 1}(\{\alpha\})$. Let $k \geq 2$. Each zero hypersurface $\mathcal{Z}_{\text {sing, } k}\left(\mathcal{I}_{k}\right)$ for given subset $\mathcal{I}_{k}$ divides the $Z C D_{k}$ in two subdomains where the function $\mathcal{Z}_{k}\left(\mathcal{I}_{k} ; \boldsymbol{x}\right)$ have definite, positive or negative, sign. The set of all $\left(\begin{array}{l}N \\ k\end{array}\right)$ hypersurfaces $\mathcal{Z}_{\text {sing, } k}\left(\mathcal{I}_{1}\right)$ with all possible partitions of $[N] \mathcal{I}_{1} \cup \mathcal{I}_{2}=[N]$ and cardinality $\# \mathcal{I}_{1}=k$ divides the $Z C D_{k}$ into a finite, say $M$, number of subdomains $Z C D_{k ; \delta}$ and inside each of them each of functions $\mathcal{Z}_{k}\left(\mathcal{I}_{k} ; \boldsymbol{x}\right)$ has definite sign.

So, one can associate with each such subdomain $Z C D_{k ; \delta}$ a set of $\left(\begin{array}{l}N \\ k\end{array}\right)$ numbers 
1 and -1 coinciding with values of sign function defined as

$$
s\left(\mathcal{I}_{1} ; \boldsymbol{x}\right):=\operatorname{sign}\left[-\sum_{\alpha \in \mathcal{I}_{1}} e^{f_{\alpha}(\boldsymbol{x})}+\sum_{\beta \in[N] \backslash \mathcal{I}_{1}} e^{f_{\beta}(\boldsymbol{x})}\right] \in\{-1,0,+1\}
$$

evaluated for each partition $\mathcal{I}_{1} \cup \mathcal{I}_{2}=[N]$ with $\# \mathcal{I}_{1}=k$ and $\boldsymbol{x} \in \mathbb{R}^{n}$. If one chooses an order for the subdomains $\left\{Z C D_{k ; \delta}\right\} \rightarrow[M]$ and for elements of $\mathcal{P}_{k}[N]$, e.g. lexicographical order, then one has the set of mappings

$$
\left(\boldsymbol{S}_{k}(\boldsymbol{x})\right)_{\tau}:=s\left(\mathcal{I}_{\tau} ; \boldsymbol{x}\right), \quad \tau=1, \ldots,\left(\begin{array}{c}
N \\
k
\end{array}\right)
$$

and

$$
\boldsymbol{S}_{k ; \delta}:=\boldsymbol{S}_{k}(\boldsymbol{x})
$$

which assigns to a subdomain $Z C D_{k ; \delta}$ a vector of $\left(\begin{array}{l}N \\ k\end{array}\right)$ components, whose $\tau$-th component is the sign of $\mathcal{Z}_{k}\left(\mathcal{I}_{\tau} ; \boldsymbol{x}\right)$ evaluated at an interior point $\boldsymbol{x} \in Z C D_{k ; \delta}$. With a slight abuse of notation, we will also use $\delta \in[M]$ to denote the corresponding $Z C D_{k ; \delta}$ with the chosen order. For example, $\boldsymbol{S}_{2 ; \delta}=(-1,1,1,1,1,-1,-1$, $-1,-1,1,1,1,1,1,1)$ where $\left\{f_{\alpha}\right\}$ are as in figure 3 and $\delta$ is the subdomain containing the point $(x, y) \equiv(2,-2)$. In the domain $\mathcal{D}_{k+}$ one has $\boldsymbol{S}_{k ; \mathcal{D}_{k+}}=(1,1,1, \ldots, 1)$. Number of signs -1 in $\boldsymbol{S}_{k ; \delta}$ varies in $Z C D_{k} \cup \mathcal{D}_{k-}$. One has

Proposition 5. The maximum number of -1 in $\boldsymbol{S}_{k ; \delta}$ at fixed $k<\frac{N}{2}$ and varying $\delta \in[M]$ is equal to $\left(\begin{array}{c}N-1 \\ k-1\end{array}\right)$. If $2 k=N$ then the number of -1 signs in $\boldsymbol{S}_{\frac{N}{2}}$ is identically equal to $\left(\begin{array}{c}2 k-1 \\ k\end{array}\right)$ on $\mathbb{R}^{n} \backslash \mathcal{Z}_{\text {sing, } \frac{N}{2}}$.

Proof. At fixed $k<\frac{N}{2}$ and for any subdomain $Z C D_{k ; \delta}$ one can consider the family

$$
\mathcal{F}_{k ; \delta-}:=\left\{\mathcal{I} \in \mathcal{P}_{k}[N]: s_{k ; \delta}(\mathcal{I})=-1\right\} .
$$

The intersection between two elements of $\mathcal{F}_{k ; \delta-}$ is non-empty. Indeed, let us assume that $\mathcal{I} \in \mathcal{F}_{k ; \delta-}$ and $\mathcal{I} \cap \mathcal{J}=\emptyset$, with $\# \mathcal{J}=k$. In particular, one has $\mathcal{J} \subseteq[N] \backslash \mathcal{I}$ and $\mathcal{I} \subseteq[N] \backslash \mathcal{J}$. This implies that

$$
\sum_{\alpha \in[N] \backslash \mathcal{J}} e^{f_{\alpha}(\boldsymbol{x})}>\sum_{\alpha \in \mathcal{I}} e^{f_{\alpha}(\boldsymbol{x})}>\sum_{\beta \in[N] \backslash \mathcal{I}} e^{f_{\beta}(\boldsymbol{x})} \geq \sum_{\beta \in \mathcal{J}} e^{f_{\beta}(\boldsymbol{x})} .
$$


Thus, $\mathcal{Z}_{k}(\mathcal{J})>0$ and $\mathcal{J} \notin \mathcal{F}_{k ; \delta-}$. Then, the family $\mathcal{F}_{k ; \delta-}$ of all $k$-subsets, $k<\frac{N}{2}$, corresponding to a -1 sign is an intersecting family, that is a family of subsets with same cardinality $k$ and pairwise non-empty intersections. Hence, Erdős-Ko-Rado theorem for intersecting family (see e.g. ${ }^{48}$ ) holds and so $\mathcal{F}_{k ; \delta-}$ has at most $\left(\begin{array}{l}N-1 \\ k-1\end{array}\right)$ elements. Moreover, this maximum is reached exactly if all elements of the family contain a certain $\alpha_{0} \in[N]$. This maximum is indeed attained. Let us consider 1-domains $D_{1-}(\alpha), \alpha \in[N]$, defined as

$$
D_{1-}(\alpha):=\left\{\boldsymbol{x} \in \mathbb{R}^{n}: \mathcal{Z}_{1}(\{\alpha\} ; \boldsymbol{x})=-e^{f_{\alpha}(\boldsymbol{x})}+\sum_{\beta \neq \alpha} e^{f_{\beta}(\boldsymbol{x})}<0\right\}, \quad \alpha=1, \ldots, N .
$$

The linearity of functions $f_{\alpha}$ assures that not all $D_{1-}(\alpha)$ are empty since the assumptions of proposition 3 are satisfied and $\vec{r}\left(t_{1}\right) \in D_{1-}\left(\alpha_{0}\right)$. If $\boldsymbol{x} \in D_{1-}(\alpha)$ then one has $\left|\mathcal{Z}_{k}(\mathcal{I} ; \boldsymbol{x})\right|=$ $\left|-\sum_{\alpha \in \mathcal{I}} e^{f_{\alpha}(\boldsymbol{x})}+\sum_{\beta \in[N] \backslash \mathcal{I}} e^{f_{\beta}(\boldsymbol{x})}\right|>e^{f_{\alpha}(\boldsymbol{x})}-\sum_{\beta \neq \alpha} e^{f_{\beta}(\boldsymbol{x})}>0$ for all $\mathcal{I} \in \mathcal{P}_{k}[N]$, so $D_{1-}(\alpha) \notin$ $\mathcal{Z}_{\text {sing, }, k}$ and all components of $\boldsymbol{S}_{k}(\boldsymbol{x})$ are not vanishing. Let us denote a subdomain $Z C D_{k ; \delta}$ such that $D_{1-}(\alpha) \cap \delta(\alpha) \neq \emptyset$ as $\delta(\alpha)$. Then, $\boldsymbol{S}_{k ; \delta(\alpha)}$ coincides with $\boldsymbol{S}_{k}(\boldsymbol{x})$, $\boldsymbol{x} \in \mathcal{D}_{1-} \cap \delta(\alpha)$. The definition of $D_{1-}(\alpha)$ implies that $\mathcal{Z}_{k}(\mathcal{I} ; \boldsymbol{x})<0$ if and only if $\alpha \in \mathcal{I}$ and the number of -1 signs in $\boldsymbol{S}_{k ; \delta(\alpha)}$ is equal to the number of $k$-subsets $\mathcal{I} \subset[N]$ containing $\alpha$, that is the maximum $\left(\begin{array}{c}N-1 \\ k-1\end{array}\right)$.

At $2 k=N$ and for any point $\boldsymbol{x} \in \mathbb{R}^{n} \backslash \mathcal{Z}_{\text {sing, } \frac{N}{2}}$ one has $\mathcal{Z}_{\frac{N}{2}}(\mathcal{I})<0$ if and only if $\mathcal{Z}_{\frac{N}{2}}([N] \backslash \mathcal{I})>0$. Since both $\mathcal{I}$ and $[N] \backslash \mathcal{I}$ have cardinality $\frac{N}{2}$, there is the same number of negative and positive terms in $\boldsymbol{S}_{\frac{N}{2}}(\boldsymbol{x})$ for all $\boldsymbol{x} \in \mathbb{R}^{n} \backslash \mathcal{Z}_{\text {sing, } \frac{N}{2}}$, that is $\left(\begin{array}{c}2 k-1 \\ k\end{array}\right)=\left(\begin{array}{c}2 k-1 \\ k-1\end{array}\right)=\frac{1}{2}\left(\begin{array}{c}2 k \\ k\end{array}\right)$

Thus, the ambient domain $\mathcal{D}_{k-}$ for each statistical $k$-amoeba is the domain of maximal instability. Previous proposition implies following

Corollary 1. One has

$$
\mathcal{D}_{k-} \subseteq \mathcal{D}_{\hat{k}-}
$$


for all $1 \leq k<\hat{k}<\frac{N}{2}$.

Proof. Let us take $\boldsymbol{x} \in \mathcal{D}_{k-}$ at $1 \leq k<\hat{k}<\frac{N}{2}$. From Erdős-Ko-Rado theorem and proposition 5 it follows that there exists $\alpha_{0} \in[N]$ such that $\mathcal{Z}_{k}(\mathcal{I} ; \boldsymbol{x})<0$ if and only if $\alpha_{0} \in \mathcal{I}$. Then, let us consider $\mathcal{J} \subset[N]$ with $\# \mathcal{J}=\hat{k}$ and $\alpha_{0} \in \mathcal{J}$. One can choose a subset $\mathcal{I}_{\mathcal{J}} \subset \mathcal{J}$ such that $\# \mathcal{I}_{\mathcal{J}}=k$ and $\alpha_{0} \in \mathcal{I}$, thus $\mathcal{Z}_{\hat{k}}(\mathcal{J} ; \boldsymbol{x})<$ $\mathcal{Z}_{k}\left(\mathcal{I}_{\mathcal{J}} ; \boldsymbol{x}\right)<0$ where last inequality holds since $\alpha_{0} \in \mathcal{I}_{\mathcal{J}}$. Then, $\mathcal{Z}_{\hat{k}}(\mathcal{J})<0$ if $\alpha_{0} \in \mathcal{J}$ and $\mathcal{Z}_{\hat{k}}(\mathcal{J})>0$ otherwise, since additional -1 signs would contradict the bound $\left(\begin{array}{l}N-1 \\ \hat{k}-1\end{array}\right)$ in proposition 5 . This means that $\boldsymbol{x} \in \mathcal{D}_{\hat{k}-}$.

Consequently one also has the chain

$$
\mathcal{D}_{1-} \subseteq \mathcal{D}_{2-} \subseteq \cdots \subseteq \mathcal{D}_{\left(\left\lceil\frac{N}{2}\right\rceil-1\right)-}
$$

which is dual with respect to (4.2). It is equivalent to

$$
\mathcal{D}_{0+} \cup Z C D_{1} \supseteq \mathcal{D}_{2+} \cup Z C D_{2} \supseteq \cdots \supseteq \mathcal{D}_{\left(\left\lceil\frac{N}{2}\right\rceil-1\right)+} \cup Z C D_{\left(\left\lceil\frac{N}{2}\right\rceil-1\right)} \cdot
$$

So, the domain of complete stability $D_{k+}$ (possible) equilibrium shrinks in transition to higher strata while the domain of instability expands. Note that there is no domain in $\mathbb{R}^{n}$ where all $\mathcal{Z}_{k}(\mathcal{I})$ are negative if $k \leq\left\lfloor\frac{N}{2}\right\rfloor$.

For each subdomain $Z C D_{k ; \delta}$ and the corresponding set $\boldsymbol{S}_{k ; \delta}$ one can introduce also its integral characteristic

$$
\overline{\boldsymbol{S}}_{k ; \delta}=\frac{1}{\left(\begin{array}{l}
N \\
k
\end{array}\right)} \cdot \sum_{\tau=1}^{\left(\begin{array}{c}
N \\
k
\end{array}\right)}\left(\boldsymbol{S}_{k ; \delta}\right)_{\tau}
$$

This quantity take values in the interval $\left[1-2 \frac{k}{N} ; 1\right], k \leq\left\lfloor\frac{N}{2}\right\rfloor$. The maximum is reached in the stability domain $\mathcal{D}_{k+}$ while the minimum $-1+2 \frac{k}{N}$ is archieved at the ambient domain $\mathcal{D}_{k-}$ of maximal instability. An absolute minimum of $\overline{\boldsymbol{S}}_{k ; \delta}$ 
equal to 0 is reached in $\mathbb{R}^{n}$ when $N$ is even and $N=2 k$. An example of the function $\overline{\boldsymbol{S}}_{k ; \delta}$ is presented in the figure 12 .

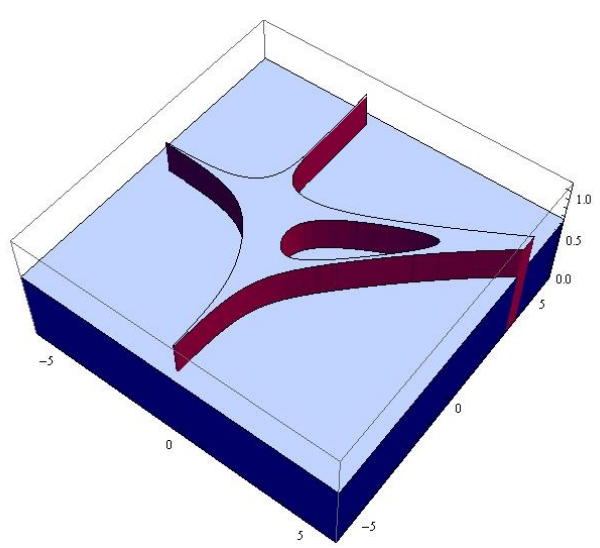

(a) $\overline{\boldsymbol{S}}_{1 ; \delta}=\frac{1}{6} \cdot \sum_{\tau=1}^{6}\left(\boldsymbol{S}_{1 ; \delta}\right)_{\tau}$.

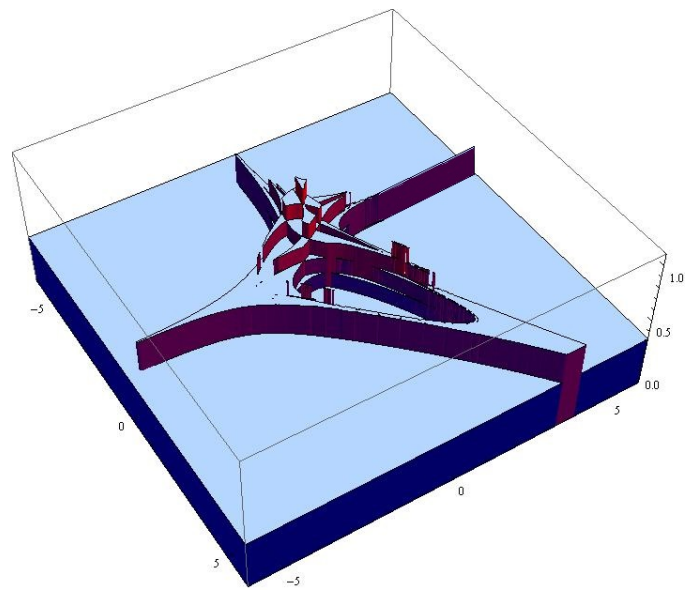

(b) $\overline{\boldsymbol{S}}_{2 ; \delta}=\frac{1}{15} \cdot \sum_{\tau=1}^{15}\left(\boldsymbol{S}_{2 ; \delta}\right)_{\tau}$.

Figure 12. $\quad \overline{\boldsymbol{S}}_{1 ; \delta}$ and $\overline{\boldsymbol{S}}_{2 ; \delta}$ in the case $f_{1} \equiv 0, f_{2} \equiv 3 x, f_{3} \equiv 3 y, f_{4} \equiv x+y+\ln 6$, $f_{5} \equiv 2 x+y+\ln 11, f_{6} \equiv x+3 y+\ln 4$.

The formula (6.10) suggests also a natural interpretation of $\overline{\boldsymbol{S}}_{k ; \delta}$. Indeed, let us view values of sign function (6.1) as two projections +1 and -1 of a "spin" associated with the subdomain $Z C D_{k ; \delta}$ and certain functions $\mathcal{Z}_{k, \tau}(\boldsymbol{x})$. So at the subdomain $Z C D_{k ; \delta}$ one has a set of $\left(\begin{array}{l}N \\ k\end{array}\right)$ "spins" with different projections. Assuming that projections associated with functions $\mathcal{Z}_{k, \tau}$ at different $\tau$ are realised with the same probability $w_{N, k}=\frac{1}{\left(\begin{array}{c}N \\ k\end{array}\right)}$ then $\overline{\boldsymbol{S}}_{k ; \delta}$ defined by (6.10) is just the mean value of spin at the subdomain $Z C D_{k ; \delta}$.

Further, one can view the collection of $\boldsymbol{S}_{k ; \delta}$ for all subdomain $Z C D_{k ; \delta}$ and domains $\mathcal{D}_{k+}, \mathcal{D}_{k-}$ as the set of states of the statistical system of $\left(\begin{array}{l}N \\ k\end{array}\right)$ spins. Considering the interaction of spins with external (magnetic) field $H$ as for the standard spin systems (see e.g. ${ }^{1,2}$ ), one defines the energy

$$
E_{k ; \delta}=-H \cdot \sum_{\tau=1}^{\left(\begin{array}{c}
N \\
k
\end{array}\right)}\left(\boldsymbol{S}_{k ; \delta}\right)_{\tau}, \quad \delta=1, \ldots, M
$$


Finally, for the partition function of the spin system one has

$$
\mathcal{Z}_{k, \text { spin }}=\sum_{\delta=1}^{M} \exp \left(-\beta H \cdot \sum_{\tau=1}^{\left(\begin{array}{c}
N \\
k
\end{array}\right)}\left(\boldsymbol{S}_{k ; \delta}\right)_{\tau}\right), \quad k=1, \ldots,\left\lfloor\frac{N}{2}\right\rfloor
$$

where $\beta$ is a parameter (say inverse of "temperature" $T$ ).

Energy $E_{k}$ has minimum at the domain $\mathcal{D}_{k+}$ and maximum in the domain $\mathcal{D}_{k-}$. Excited transition states are associated with subdomains of $Z C D_{k}$.

Introducing interaction between spins of the form $E_{k ; \delta \text {,int }}=\gamma \cdot \sum_{\tau, \nu=1}^{\left(\begin{array}{c}N \\ k\end{array}\right)}\left(\boldsymbol{S}_{k ; \delta}\right)_{\tau} \cdot\left(\boldsymbol{S}_{k ; \delta}\right)_{\nu}$, one gets a partition function of the Ising type model.

\section{Tropical limit and tropical zeros}

The amoebas viewed at large distance are essentially the sets of thinning tentacles which become certain piecewise linear objects in the tropical limit for algebraic amoebas, see e.g. ${ }^{42,49}$. Such images of statistical $k$-amoebas are associated with the limiting behaviour at large functions $f_{\alpha}(\boldsymbol{x})$ in the partition function (1.2). In the case of linear functions $f_{\alpha}(\boldsymbol{x})$ as in (2.1) there are different ways to realise such a limit. The first one is to consider large values of the variables $x_{i}$ introducing slow variables $\tilde{x}_{i}:=\varepsilon \cdot x_{i}, i=1, \ldots, n$ with $\varepsilon \rightarrow 0$. For the $k$-th stratum the functions $\mathcal{Z}_{k}\left(\mathcal{I}_{\tau} ; \boldsymbol{x}\right)$ at $\varepsilon \rightarrow 0$ are the superpositions of highly singular terms and the corresponding hypersurfaces are defined as

$$
\sum_{\alpha=1}^{N} g_{\mathcal{I}_{\tau}(\alpha)} \exp \left(\frac{1}{\varepsilon} \cdot \sum_{i=1}^{n} a_{\alpha i} \tilde{x}_{i}\right)=0, \quad \tau=1, \ldots,\left(\begin{array}{c}
N \\
k
\end{array}\right)
$$

as $\varepsilon \rightarrow 0$. For the first stratum $(k=1)$ equations (7.1) are of the form

$$
\exp \left(\frac{1}{\varepsilon} \cdot \sum_{i=1}^{n} a_{\alpha i} \tilde{x}_{i}\right)=\sum_{\beta \neq \alpha} \exp \left(\frac{1}{\varepsilon} \cdot \sum_{i=1}^{n} a_{\beta i} \tilde{x}_{i}\right)
$$

and in the limit $\varepsilon \rightarrow 0$ one gets the set of hyperplanes in $\mathbb{R}^{n}$ given by

$$
\sum_{i=1}^{n} a_{\alpha i} \tilde{x}_{i}=\max _{\beta \neq \alpha}\left\{\sum_{i=1}^{n} a_{\beta i} \tilde{x}_{i}\right\} .
$$


All these hyperplanes pass through the origin $\tilde{x}_{i}=0, i=1, \ldots, n$. They are the tropical limit of the ideal statistical hypersurfaces considered $\mathrm{in}^{37}$.

For higher strata and each partition $\left(\mathcal{I}_{1}, \mathcal{I}_{2}\right)$ the limit $\varepsilon \rightarrow 0$ of equations (3.4), (3.5) is given by the set of hyperplanes

$$
\max _{\alpha \in \mathcal{I}_{1}}\left\{\sum_{i=1}^{n} a_{\alpha i} \tilde{x}_{i}\right\}=\max _{\beta \in \mathcal{I}_{2}}\left\{\sum_{i=1}^{n} a_{\beta i} \tilde{x}_{i}\right\} .
$$

The second way to realise the limit $f_{\alpha} \rightarrow \infty$, more close to the standard tropical limit in algebraic geometry ${ }^{42,49}$, is to make the parameter $b_{\alpha}$ in $f_{\alpha}$ large too, i.e. to consider the limit $x_{i}=\frac{\tilde{x}_{i}}{\varepsilon}, b_{\alpha}=\frac{\tilde{b}_{\alpha}}{\varepsilon}$, with finite $\tilde{x}_{i}, \tilde{b}_{\alpha}$ and $\varepsilon \rightarrow 0$. In this case the tropical limit of the hypersurfaces (3.4), (3.5) is given by

$$
\max _{\alpha \in \mathcal{I}_{1}}\left\{\tilde{b}_{\alpha}+\sum_{i=1}^{n} a_{\alpha i} \tilde{x}_{i}\right\}=\max _{\beta \in \mathcal{I}_{2}}\left\{\tilde{b}_{\beta}+\sum_{i=1}^{n} a_{\beta i} \tilde{x}_{i}\right\} .
$$

Now the hyperplanes (7.5) do not pass, in general, through the origin $\tilde{x}_{i}=0$, $i=1, \ldots, n$.

The third way is to keep variables $x_{i}$ finite, but to send to infinity the parameters $a_{\alpha i}$ and $b_{\alpha}$ as $a_{\alpha i}=\frac{\tilde{a}_{\alpha i}}{\varepsilon}, b_{\alpha}=\frac{\tilde{b}_{\alpha}}{\varepsilon}$, with $\varepsilon \rightarrow 0$ and finite $\tilde{a}_{\alpha i}, \tilde{b}_{\alpha}$. Such a limit of hypersurfaces $(3.4),(3.5)$ is given by the set of hyperplanes defined by equations

$$
\max _{\alpha \in \mathcal{I}_{1}}\left\{\tilde{b}_{\alpha}+\sum_{i=1}^{n} \tilde{a}_{\alpha i} x_{i}\right\}=\max _{\beta \in \mathcal{I}_{2}}\left\{\tilde{b}_{\beta}+\sum_{i=1}^{n} \tilde{a}_{\beta i} x_{i}\right\} .
$$

Equations (7.5) and (7.6) are related via exchange $a_{\alpha i} \leftrightarrow \tilde{a}_{\alpha i}, \tilde{x}_{i} \leftrightarrow x_{i}$ keeping in both cases the product $a_{\alpha i} x_{i} \sim \frac{1}{\varepsilon}$.

For different strata the sets of equations (7.5) or (7.6), defining the tropical limit of hypersurfaces (3.4), (3.5) are quite different. However, one has

Proposition 6. In the tropical limits considered above, zeros loci of $\mathcal{Z}_{k, \text { trop }}(\mathcal{I})$ given by equations (7.5) or (7.6) are the same for all strata. All domains $Z C D_{k}$ collapse into a single set of piecewise hyperplanes given e.g. by equations (7.5) or (7.6) for the first stratum $\mathcal{Z}_{\text {sing, } 1 \text {. }}$ 
Proof. Let us denote $\mathcal{I}_{1}:=\mathcal{I}$ and $\mathcal{I}_{2}:=[N] \backslash \mathcal{I}$. The equation $\mathcal{Z}_{k}\left(\mathcal{I}_{1} ; \boldsymbol{x}\right)=0$, $\# \mathcal{I}_{1}=k$, is equivalent to $\sum_{\alpha \in \mathcal{I}_{1}} e^{f_{\alpha}(\boldsymbol{x})}=\sum_{\beta \in \mathcal{I}_{2}} e^{f_{\beta}(\boldsymbol{x})}$. In term of slow variables it becomes $\sum_{\alpha \in \mathcal{I}_{1}} \exp \left(\frac{f_{\alpha}(\boldsymbol{x})}{\varepsilon}\right)=\sum_{\beta \in \mathcal{I}_{2}} \exp \left(\frac{f_{\beta}(\boldsymbol{x})}{\varepsilon}\right)$. Let us take $\bar{\alpha}_{i} \in \mathcal{I}_{i}$ such that $f_{\bar{\alpha}_{i}}(\boldsymbol{x})=\max _{\alpha \in \mathcal{I}_{i}}\left\{f_{\alpha}(\boldsymbol{x})\right\}, i \in\{1,2\}$. Then, previous equation is equivalent to

$$
\exp \frac{f_{\bar{\alpha}_{1}}(\boldsymbol{x})}{\varepsilon} \cdot\left[\sum_{\beta \in \mathcal{I}_{1}} \exp \frac{f_{\beta}(\boldsymbol{x})-f_{\bar{\alpha}_{1}}(\boldsymbol{x})}{\varepsilon}\right]=\exp \frac{f_{\bar{\alpha}_{2}}(\boldsymbol{x})}{\varepsilon} \cdot\left[\sum_{\gamma \in \mathcal{I}_{2}} \exp \frac{f_{\gamma}(\boldsymbol{x})-f_{\bar{\alpha}_{2}}(\boldsymbol{x})}{\varepsilon}\right] .
$$

Both the factors in square bracket in (7.7) lie in the interval $[1, N-k]$ independently on $\varepsilon \in \mathbb{R}_{+}$. Hence they are finite and non-vanishing. Thus, exp $\frac{f_{\mathcal{I}_{1}}(\boldsymbol{x})-f_{\mathcal{I}_{2}}(\boldsymbol{x})}{\varepsilon}$ lies in $\left[\frac{1}{N-k} ; N-k\right]$ for all $\varepsilon \in \mathbb{R}_{+}$. Considering the limit $\varepsilon \rightarrow 0$ one gets

$$
\max _{\alpha \in \mathcal{I}_{1}}\left\{f_{\alpha}(\boldsymbol{x})\right\}=\max _{\beta \in \mathcal{I}_{2}}\left\{f_{\beta}(\boldsymbol{x})\right\}
$$

Note that $\bar{\alpha}_{1} \neq \bar{\alpha}_{2}$ since they belong to different parts of the partition. For any $\gamma \in[N], \gamma \in \mathcal{I}_{i}$ for exactly one $i \in\{1,2\}$, so $f_{\gamma}(\boldsymbol{x}) \leq \max _{\alpha \in \mathcal{I}_{1}}\left\{f_{\alpha}(\boldsymbol{x})\right\}=f_{\bar{\alpha}_{i}}(\boldsymbol{x})$. Thus, $f_{\bar{\alpha}_{1}}(\boldsymbol{x})=f_{\bar{\alpha}_{2}}(\boldsymbol{x})=\max _{\gamma \in[N]}\left\{f_{\gamma}(\boldsymbol{x})\right\}$ so the maximum $\max _{\gamma \in[N]}\left\{f_{\gamma}(\boldsymbol{x})\right\}$ is attained at least twice, once for each index $i \in\{1,2\}$ of $\mathcal{I}_{i}$. Considering all such partitions with $\# \mathcal{I}=k$, one gets the union of all these tropical loci. This is the set of all points $\boldsymbol{x} \in \mathbb{R}^{n}$ such that maximum of $\left\{f_{1}(\boldsymbol{x}), \ldots, f_{N}(\boldsymbol{x})\right\}$ is attained at least twice and it is independent of the stratum $k$ considered.

So, in the tropical limit the statistical amoebas collapse into the $(n-1)$ dimensional objects $\mathcal{A}_{\text {trop }}$ formed by pieces of hyperplanes and the maximal instability domais $\mathcal{D}_{k-}$ expand to the almost whole space $\mathbb{R}^{n}$, namely to $\mathbb{R}^{n} \backslash \mathcal{A}_{\text {trop }}$. Points of the piecewise hyperplanes $\mathcal{A}_{\text {trop }}$ are tropical zeros of partition function.

These different kinds of tropical limit provide different structures for the same underlying model. For example, the tropical limit of the first kind (7.4) highlights the degree 1 homogeneous part of linear functions $f_{\alpha}$. More in general, it gives the dominant homogeneous parts of functions $f_{\alpha}$ and can be applied in the study 
of emergence of degenerate metrics from tropical limit, see e.g. ${ }^{37}$. An advantage of tropical limit of the first kind is that it has a rather simple geometry.

Lemma 3. If $f_{\alpha}$ are $N$ real functions then $\sum_{\alpha=1}^{N} e^{\lambda \cdot f_{\alpha}(\boldsymbol{x})} \leq\left(\sum_{\alpha=1}^{N} e^{f_{\alpha}(\boldsymbol{x})}\right)^{\lambda}$ for all $\lambda \geq$ 1 .

Proof. For all $\lambda \geq 1$ one has

$$
0<\left(\frac{e^{f_{\alpha}(\boldsymbol{x})}}{\sum_{\beta} e^{f_{\beta}(\boldsymbol{x})}}\right)^{\lambda} \leq \frac{e^{f_{\alpha}(\boldsymbol{x})}}{\sum_{\beta} e^{f_{\beta}(\boldsymbol{x})}}<1, \quad \alpha \in[N],
$$

which implies

$$
0<\sum_{\alpha=1}^{N}\left(\frac{e^{f_{\alpha}(\boldsymbol{x})}}{\sum_{\beta} e^{f_{\beta}(\boldsymbol{x})}}\right)^{\lambda} \leq \sum_{\alpha=1}^{N} \frac{e^{f_{\alpha}(\boldsymbol{x})}}{\sum_{\beta} e^{f_{\beta}(\boldsymbol{x})}}=1 \Rightarrow \sum_{\alpha=1}^{N} e^{\lambda f_{\alpha}(\boldsymbol{x})} \leq\left(\sum_{\alpha=1}^{N} e^{f_{\alpha}(\boldsymbol{x})}\right)^{\lambda}
$$

Proposition 7. Connected components of the complement of the tropical graph of the first kind are unbounded. For homogeneous functions, connected components of $\mathcal{D}_{1-}$ are unbounded too.

Proof. Given $N$ linear functions $f_{1}, \ldots, f_{N}$, let $\varphi_{\alpha}(\boldsymbol{x})=f_{\alpha}(\boldsymbol{x})-f_{\alpha}(\mathbf{0})$ be the 1homogeneous part of $f_{\alpha}, \Delta_{1-}(\alpha):=\left\{\boldsymbol{y} \in \mathbb{R}^{n}: e^{\varphi_{\alpha}(\boldsymbol{y})}>\sum_{\beta \neq \alpha} e^{\varphi_{\beta}(\boldsymbol{y})}\right\}$ be the instability domain where $\varphi_{\alpha}$ dominates and $\Delta_{1-}^{\text {trop }}(\alpha):=\left\{\boldsymbol{y} \in \mathbb{R}^{n}: \varphi_{\alpha}(\boldsymbol{y})>\max _{[N] \backslash\{\alpha\}}\left\{\varphi_{\beta}(\boldsymbol{y})\right\}\right\}$ be the tropical limit of $\Delta_{1-}(\alpha)$. In particular, it easily follows from the definitions that $\Delta_{1-}(\alpha) \subseteq \Delta_{1-}^{\text {trop }}(\alpha)$. From (7.4), $\left\{f_{\alpha}\right\}$ and $\left\{\varphi_{\alpha}\right\}$ have the same tropical limit of the first kind, so we focus on the latter set of functions. If $\boldsymbol{x} \in \Delta_{1-}(\alpha)$ and $\lambda \geq 1$ then

$$
e^{\varphi_{\alpha}(\lambda \boldsymbol{x})}=\left(e^{\varphi_{\alpha}(\boldsymbol{x})}\right)^{\lambda}>\left(\sum_{\beta \neq \alpha} e^{\varphi_{\beta}(\boldsymbol{x})}\right)^{\lambda} \geq \sum_{\beta \neq \alpha} e^{\lambda \varphi_{\beta}(\boldsymbol{x})}=\sum_{\beta \neq \alpha} e^{\varphi_{\beta}(\lambda \boldsymbol{x})}
$$

where the second inequality follow from lemma 3 applied to homogeneous functions $\varphi_{\beta}, \beta \neq \alpha$. Hence $\lambda \cdot \boldsymbol{x} \in \Delta_{1-}(\alpha)$ for all $\boldsymbol{x} \in \Delta_{1-}(\alpha)$ and $\lambda \geq 1$. In the same way 
one can show that $\lambda \cdot \boldsymbol{x} \in \Delta_{1-\text {,trop }}(\alpha)$ for all $\boldsymbol{x} \in \Delta_{1-\text {,trop }}(\alpha)$ and $\lambda \geq 1$. So, let $\mathcal{C}$ (respectively, $\mathcal{C}^{\star}$ ) be a connected component of $\Delta_{1-}(\alpha)$ (respectively, of $\Delta_{1-}^{\text {trop }}(\alpha)$ ) and choose $\boldsymbol{x} \in \mathcal{C}$ (respectively, $\boldsymbol{x}^{\star} \in \mathcal{C}^{\star}$ ). One has $\{\lambda \cdot \boldsymbol{x}: \lambda \geq 1\} \subseteq \mathcal{C}$ since the ray $\{\lambda \cdot \boldsymbol{x}: \lambda \geq 1\}$ is a connected subset of $\Delta_{1-}(\alpha)$ intersecting $\mathcal{C}$ and $\mathcal{C}$ is maximal among connected subsets of $\Delta_{1-}(\alpha)$. Similarly, $\left\{\lambda \cdot \boldsymbol{x}^{\star}: \lambda \geq 1\right\} \subseteq \mathcal{C}^{\star}$. Thus both $\mathcal{C}$ and $\mathcal{C}^{\star}$ are unbounded since they contain an unbounded subset.

Thus, tropical limit of the first kind has simple topological properties. For example, in two-dimensional case, the result of proposition 7 means a trivial homotopy for the resulting tropical graph.

It is worth mentioning that terms in (7.4) coincide with $f_{\alpha}(\boldsymbol{x})$ if $f_{\alpha}(\mathbf{0})=0$ for all $\alpha \in[N]$. Homogeneous linear functions $f_{\alpha}(\boldsymbol{x}) \equiv \sum_{i=1}^{n} \kappa_{\alpha}^{i} x_{i}$ with real distinct parameters $\kappa_{1}<\cdots<\kappa_{N}$ represent a particular example. These functions arise in the study of Wronskian soliton solutions of KP II equation where $e^{f_{\alpha}(x)}$ are special solutions of the heat hierarchy. If one considers the tropical limit of the second kind (7.5) instead of (7.4), then the resulting object has a more refined structure and many combinatorial properties (see e.g. ${ }^{50}$ ).

Tropical limits discussed above are quite meaningful in the statistical physics of macrosystems. Tropical limit of free energy considered in ${ }^{51}$ corresponds to $n=1$, $\tilde{x}_{1}=\frac{1}{k_{B} T}, a_{\alpha 1}=-E_{\alpha}, b_{\alpha}=\frac{S_{\alpha}}{k_{B}}, \varepsilon=k_{B}$ where $T$ is the temperature, $\left\{E_{\alpha}\right\}$ is the energy spectrum, $\exp \left(\frac{S_{\alpha}}{k_{B}}\right)$ are degenerations of energy levels and $k_{B}$ is the Boltzmann constant.

One can consider also more complicated situations when some of the products $a_{\alpha i} \cdot x_{i}$ remain finite, for instance, when $x_{i_{0}}=\frac{\tilde{x}_{i_{0}}}{\varepsilon}$ and $a_{\alpha i_{0}}=\varepsilon \cdot \tilde{a}_{\alpha i_{0}}$. In such a case the product $a_{\alpha i_{0}} \cdot x_{i_{0}}$ does not contribute in the limit $\varepsilon \rightarrow 0$ and the corresponding equation (7.4), or (7.5), will not contain the variable $\tilde{x}_{i_{0}}$. So in the tropical limit the zero locus is a piecewise hyperplane of cylindrical type.

Such non-uniform scaling behaviour of the variables $x_{i}$ or parameters $a_{\alpha i}$ and 
its connections with the multiscale tropical limit will be discussed elsewhere.

\section{Conclusion}

In this paper partition functions (1.2) with linear $f_{\alpha}(\boldsymbol{x})$ have been studied. The case of nonlinear functions $f_{\alpha}(\boldsymbol{x})$ is of great interest too. Many general properties of singular sectors described above, e.g. stratification of statistical $k$-amoebas, remain unchanged for more general polynomial functions $f_{\alpha}(\boldsymbol{x})$. Specifically, for polynomials $f_{\alpha}(\boldsymbol{x})$ the set of roots (4.4) is finite and at least one $\mathcal{D}_{1-}(\alpha)$ in (6.6) is not empty. These hypotheses are crucial for propositions as 3, 5 and corollary 1 to be valid. Proposition 7 can be generalized to polynomials by considering their degree $d$ homogeneous parts, where $d:=\max _{\alpha \in[N]}\left\{\operatorname{deg} f_{\alpha}\right\}<\infty$. Figure 13 presents an example of such a type.

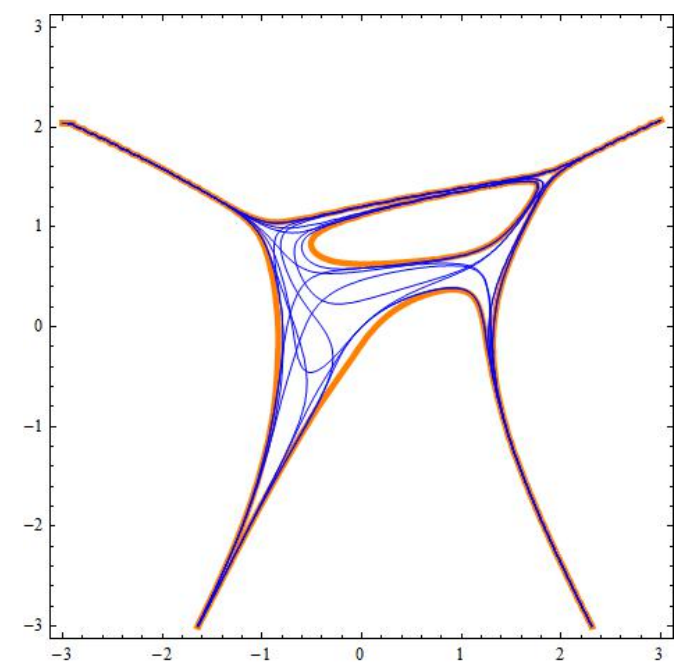

Figure 13. Stratification of $\mathcal{Z}_{\text {sing }, 2}$ (blue) and $\mathcal{Z}_{\text {sing }, 1}$ (orange) in a nonlinear polynomial case: $f_{1} \equiv 0, f_{2} \equiv 3 x^{2}, f_{3} \equiv 3 y^{3}, f_{4} \equiv x+x y+\ln 6, f_{5} \equiv 2 x+y^{2}+\ln 11, f_{6} \equiv$ $x+3 y+x y+\ln 4$.

However for general nonlinear functions situation is quite different. An example 
with non-polynomial functions

$$
f_{\alpha}(\boldsymbol{x}) \equiv\left\{\begin{array}{cc}
c_{\alpha} \cdot \eta\left(\|\boldsymbol{x}\|-\frac{\alpha+1999}{1000}\right) & \frac{\alpha+1999}{1000}>\|\boldsymbol{x}\| \\
d_{\alpha} \cdot \eta\left(\|\boldsymbol{x}\|-\frac{\alpha+1999}{1000}\right), & \|\boldsymbol{x}\| \geq \frac{\alpha+1999}{1000}
\end{array}\right.
$$

where $\eta(z)=\left\{\begin{array}{c}1-\exp \left(-\frac{z^{2}}{1-z^{2}}\right), \quad 1>|z| \\ 1, \quad|z| \geq 1\end{array},\left(c_{1}, d_{1}\right)=(\ln 20, \ln 8), \quad\left(c_{2}, d_{2}\right)=\right.$ $(\ln 20, \ln 2)$ and $\left(c_{\alpha}, d_{\alpha}\right)=(\ln 2, \ln 2), \alpha=3, \ldots, 10$, is shown in figure 14. Let us consider $S_{k}(\boldsymbol{x}):=C_{k}^{10} \cdot \overline{\boldsymbol{S}}_{k}(\boldsymbol{x})$ at $k=3,4$. At $\|\boldsymbol{x}\| \leq 1$ one has $\mathcal{Z}_{k}(\mathcal{I} ; \boldsymbol{x})<0$ iff $\{1,2\} \subset \mathcal{I}$. Thus $S_{k}(\boldsymbol{x})=\left(\begin{array}{c}10 \\ k\end{array}\right)-2 \cdot\left(\begin{array}{c}8 \\ k-2\end{array}\right)$. At $1 \leq\|\boldsymbol{x}\|<2 S_{k}(\boldsymbol{x})$ is not decreasing. At $\|\boldsymbol{x}\| \geq 2$ one has $\mathcal{Z}_{3}(\mathcal{I} ; \boldsymbol{x})>0$ for all $\mathcal{I} \in \mathcal{P}_{3}[10]$, then $S_{3}(\boldsymbol{x})=C_{3}^{10}$. On the other hand, at $\|\boldsymbol{x}\| \geq 4$ one has $\mathcal{Z}_{4}(\mathcal{I} ; \boldsymbol{x})<0$ iff $1 \in \mathcal{I}$, hence $S_{4}(\mathcal{I} ; \boldsymbol{x})$ has a minimum according to Erdos-Ko-Rado theorem ${ }^{48}$. In conclusion, $\min _{\boldsymbol{x}} S_{3}(\boldsymbol{x})=\left(\begin{array}{c}10 \\ 3\end{array}\right)-2 \cdot\left(\begin{array}{l}8 \\ 1\end{array}\right)=104$ is attained only if $\|\boldsymbol{x}\|<2$. Vice versa, $\min _{\boldsymbol{x}} S_{4}(\boldsymbol{x})=\left(\begin{array}{c}10 \\ 4\end{array}\right)-2 \cdot\left(\begin{array}{l}9 \\ 3\end{array}\right)=42$ is attained only if $\|\boldsymbol{x}\|>2$. In particular, $\mathcal{D}_{3-} \nsubseteq \mathcal{D}_{4-}$.

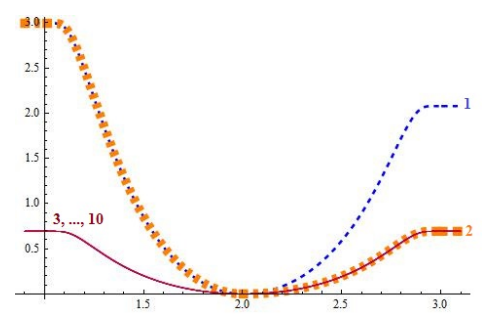

(a)Functions $f_{\alpha}(\boldsymbol{x})$, $\alpha=1, \ldots, 10$, in $(8.1)$.

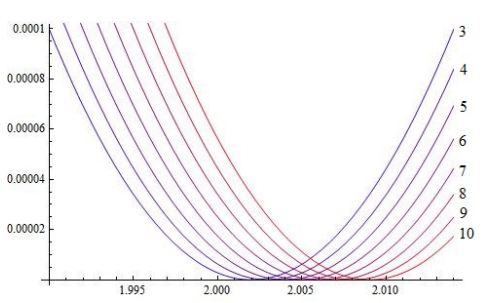

(b)Detail of functions $f_{\alpha}(\boldsymbol{x})$ in (8.1), $\alpha=3, \ldots, 10$.

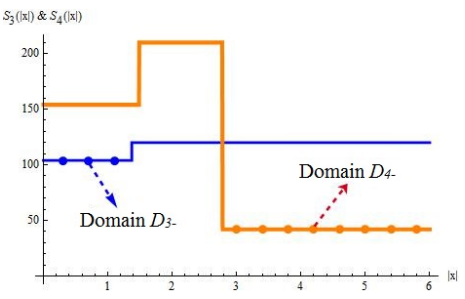

(c)Instability domain $\mathcal{D}_{3-}$ and $\mathcal{D}_{4-}$ are highlighted.

Figure 14. A non-polynomial case when chain stratification of instability domains fails.

Singular sectors of partition functions (1.2) with nonlinear $f_{\alpha}(\boldsymbol{x})$ will be considered in a separate publication. 


\section{References}

${ }^{1}$ L. D. Landau and E. M. Lifschitz, Statistical Physics, Course of Theoretical Physics, Part 1, Vol. 5 (Butterworth-Heinemann, 1980).

${ }^{2}$ K. Huang, Statistical mechanics (New York: John Wiley \& Sons, 1963).

${ }^{3}$ C. N. Yang and T. D. Lee, "Statistical theory of equations of state and phase transitions. I. Theory of condensation", Phys. Rev. 87(3), 404-409 (1952).

${ }^{4}$ T. D. Lee and C. N. Yang, "Statistical theory of equations of state and phase transitions. II. Lattice gas and Ising model", Phys. Rev., 87(3),410-419 (1952).

${ }^{5}$ M. E. Fisher, In Lectures in Theoretical Physics, Vol. 7C, Chap. 1 (W. E. Brittin (Ed.), Boulder: University of Colorado Press, 1965)

${ }^{6}$ P. J. Kortman and R. B. Griffiths, "Density of Zeros on the Lee-Yang Circle for Two Ising Ferromagnets", Phys. Rev. Lett. 27(21), 1439-1442 (1971).

${ }^{7}$ M. Suzuki and M. E. Fisher, "Zeros of the partition function for the Heisenberg, ferroelectric, and general Ising models", J. Math. Phys. 12(2), 235-246, (1971)

${ }^{8}$ D. Ruelle, "Some remarks on the location of zeroes of the partition function for lattice systems", Comm. Math. Phys. 31, 265-277 (1973).

${ }^{9}$ B. Derrida, "Random-energy model: An exactly solvable model of disordered systems", Phys. Rev. B. 24(5), 2613-2626 (1981).

${ }^{10}$ E. H. Lieb and A. D. Sokal, "A general Lee-Yang theorem for one-component and multicomponent ferromagnets", Commun. Math. Phys. 80(2), 153-179 (1981).

${ }^{11}$ R. B. Pearson, "Partition function of the Ising model on the periodic $4 \times 4 \times 4$ lattice", Phys. Rev. B 26(11), 6285-6290 (1982).

${ }^{12}$ B. Derrida, L. De Seze and C. Itzykson, "Fractal structure of zeros in hierarchical models", J. Stat. Phys. 33(3), 559-569 (1983).

${ }^{13} \mathrm{C}$. Borgs and R. Kotecký, "A rigorous theory of finite-size scaling at first-order phase transitions" J. Stat. Phys. 61(1-2), 79-119 (1990).

${ }^{14}$ M. Biskup, C. Borgs, J. T. Chayes, L. J. Kleinwaks, R. Kotecký, "Partition 
function zeros at first-order phase transitions: A general analysis" Commun. Math. Phys. 251, 79-131 (2004).

${ }^{15}$ B.-B. Wei and R.-B. Liu, "Lee-Yang zeros and critical times in decoherence of a probe spin coupled to a bath" Phys. Rev. Lett. 109(18), (2012).

${ }^{16}$ T. Obuchi and K. Takahashi, "Dynamical singularities of glassy systems in a quantum quench", Phys. Rev. E 86(5), (2012).

${ }^{17}$ B.-B. Wei, S.-W. Chen, H.-C. Po, R.-B. Liu, "Phase transitions in the complex plane of physical parameters", Sci. Rep. 4, (Nature Publishing Group, 2014).

${ }^{18}$ X. Peng, H. Zhou, B.-B. Wei, J. Cui, J. Du, R.-B. Liu, "Experimental observation of Lee-Yang zeros", Phys. Rev. Lett. 114(1), (2015).

${ }^{19}$ J. S. Langer, "Statistical theory of the decay of metastable states", Ann. Phys. 54(2), 258-275 (1969).

${ }^{20}$ C. M. Newman and L. S. Schulman, "Complex free energies and metastable lifetimes", J. Stat. Phys. 23(2), 131-148 (1980).

${ }^{21}$ G. Parisi, "A sequence of approximated solutions to the SK model for spin glasses", J. Phys. A: Math. Gen. 13(4), L115-121 (1980).

${ }^{22}$ M. Mezard, G. Parisi and M. A. Virasoro, Spin glass theory and beyond, (Singapore: World Scientific, 1987).

${ }^{23}$ Y. Ozeki Y and H. Nishimori, "Distribution of Yang-Lee Zeros of the $\pm J$ Ising Model", J. Phys. Soc. Jpn. 57(3), 1087-1093 (1988).

${ }^{24}$ B. Derrida, "The zeroes of the partition function of the random energy model" Physica A 177(1-3), 31-37 (1991).

${ }^{25} \mathrm{G}$. Bhanot and J. Lacki, "Partition function zeros and the three-dimensional Ising spin glass", J. Stat. Phys. 71(1-2), 259-267 (1993).

${ }^{26}$ Y. Matsuda, H. Nishimori and K. Hukushima, "The distribution of Lee-Yang zeros and Griffiths singularities in the $\pm J$ model of spin glasses", J. Phys. A: Math. Theor. 41(32), 324012 (2008).

${ }^{27}$ T. Obuchi and K. Takahashi, "Partition-function zeros of spherical spin glasses 
and their relevance to chaos", J. Phys. A: Math. Theor. 45(12), 125003, (2012).

${ }^{28}$ R. P. Feynman, "Negative probability", in Quantum implications: essays in honour of David Bohm, 235-248 (1987).

${ }^{29}$ W. D. Blizard, "Negative membership", Notre Dame J. Formal Logic 31(3), 346-368 (1990).

${ }^{30} \mathrm{M}$. Burgin, "Interpretations of negative probabilities", arXiv preprint, arXiv: 1008.1287 .

${ }^{31}$ K. Purbhoo, "A Nullstellensatz for amoebas", Duke. Math. J. 141(3), 407-445 (2008)

${ }^{32}$ D. Y. Pochekutov and A. K. Tsikh, "On the Asymptotics of Laurent Coefficients and its Application in Statistical Mechanics", J. Siberian Fed. Univ.: Math. Phys. 2(4), 483-493 (2009).

${ }^{33} \mathrm{M}$. Kapranov, "Thermodynamics and the moment map", arXiv preprint, arXiv:1108.3472(2011).

${ }^{34}$ M. Passare, D. Pochekutov, A. Tsikh, "Amoebas of complex hypersurfaces in statistical thermodynamics" Math. Phys. Anal. Geom. 16(1), 89-108 (2012).

${ }^{35} \mathrm{M}$. Adler and P. Van Moerbeke, "Completely integrable systems, Euclidean Lie algebras, and curves", Adv. Math. 38(3), 267-317 (1980).

${ }^{36}$ L. Casian and Y. Kodama, "Toda lattice, cohomology of compact Lie groups and finite Chevalley groups", Invent. Math. 165(1), 163-208 (2006).

${ }^{37}$ M. Angelelli and B. Konopelchenko, "Geometry of the basic statistical physics mapping", J. Phys. A: Math. Theor. 49(38), 385202 (2016).

${ }^{38}$ I. M. Gelfand, M. M. Kapranov and A. V. Zelevinsky, Discriminants, Resultants, and Multidimensional Determinants, Modern Birkhäuser Classics (Boston, MA: Birkhäuser, 2008).

${ }^{39}$ G. Mikhalkin, "Real algebraic curves, the moment map and amoebas", Ann. Math. 151(1), 309-326 (2000).

${ }^{40}$ M. Forsberg, M. Passare and A. Tsikh, "Laurent determinants and arrangements 
of hyperplane amoebas", Adv. Math. 151(1), 45-70 (2000).

${ }^{41}$ T. Theobald, "Computing amoebas", Exp. Math. 11(4), 513-526 (2002).

${ }^{42}$ G. Mikhalkin, "Amoebas of algebraic varieties and tropical geometry", in Different faces of geometry, Int. Math. Ser. Vol. 3, 257-300 (Eds. S. Donaldson, Y. Eliashberg and M. Gromov, Springer SBM, 2004).

${ }^{43}$ N. A. Bushueva and A. K. Tsikh AK, "On amoebas of algebraic sets of higher codimension", Proc. Steklov Inst. Math. 279(1), 52-63 (2012).

${ }^{44}$ M. Nisse and M. Passare, "Amoebas and coamoebas of linear spaces", arXiv preprint, arXiv:1205.2808 (2012).

${ }^{45}$ G. Mikhalkin, "Amoebas of half-dimensional varieties", arXiv preprint, arXiv: $1412.4658 \mathrm{v} 2(2015)$.

${ }^{46}$ G. Mikhalkin, "Geometry of amoebas", Lecture notes, Inst. Henri Poincaré (2013).

${ }^{47}$ R. Kenyon, A. Okounkov and S. Sheffield, "Dimers and amoebae", Ann. Math. 163(3), 1019-1056 (2006).

${ }^{48}$ P. Erdős, C. Ko and R. Rado, "Intersection theorems for systems of finite sets" Q. J. Math. 12(1), 313-320 (1961)

${ }^{49}$ D. Maclagan and B. Sturmfels, "Introduction to Tropical Geometry", Graduate Studies in Mathematics Vol. 161 (Providence, RI: Am. Math. Soc., 2015).

${ }^{50}$ Y. Kodama and L. Williams, "The Deodhar decomposition of the Grassmannian and the regularity of KP solitons", Advances in Mathematics 244, 979-1032 (2013).

${ }^{51}$ M. Angelelli and B. Konopelchenko, "Tropical Limit in Statistical Physics", Phys. Lett. A 379(24-25), 1497-1502 (2015). 